\title{
Identification and validation of a novel signature for prediction the prognosis and immunotherapy benefit in bladder cancer
}

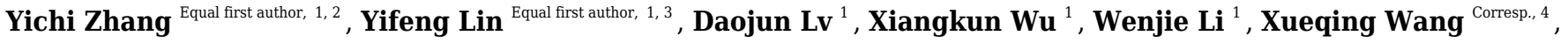 \\ Dongmei Jiang ${ }^{\text {Corresp. } 5}$ \\ 1 \\ 1 Department of Urology, the First Affiliated Hospital of Guangzhou Medical University, Guangzhou, China \\ 2 Nanshan School, Guangzhou Medical University, Guangzhou, Guangdong, China \\ 3 Department of Urology, Meizhou Hospital of Traditional Chinese Medicine, Meizhou, China \\ 4 Department of Ultrasound, Shantou Central Hospital, Shantou, Guangdong, China \\ 5 Department of Pathology, the First Affiliated Hospital of Guangzhou Medical University, Guangzhou, Guangzhou, China \\ Corresponding Authors: Xueqing Wang, Dongmei Jiang \\ Email address: m13414039585_1@163.com, 730245768@qq.com
}

Background: Bladder cancer $(\mathrm{BC})$ is a common urinary tract system tumor with high recurrence rate and different populations show distinct response to immunotherapy. Novel biomarkers that can accurately predict prognosis and therapeutic responses are urgently needed. Here, we aim to identify a novel prognostic and therapeutic responses immune-related gene signature of BC through a comprehensive bioinformatics analysis.

Methods: The robust rank aggregation was conducted to integrate differently expressed genes (DEGs) in datasets of the Cancer Genome Atlas (TCGA) and the gene expression omnibus (GEO). Lasso and Cox regression analyses were performed to formulate a novel mRNA signature that could predict prognosis of BC patients. Subsequently, the prognostic value and predictive value of the signature was validated with two independent cohorts GSE13507 and IMvigor210. Finally, quantitative Real-time PCR (qRT-PCR) analysis was conducted to determine the expression of mRNAs in BC cell lines (UM-UC-3, EJ-1, SW780 and T24).

Results: We built a signature comprised the eight mRNAs: CNKSR1, COPZ2, CXorf57, FASN, PCOLCE2, RGS1, SPINT1 and TPST1. Our prognostic signature could be used to stratify BC population into two risk groups with distinct immune profile and responsiveness to immunotherapy. The results of qRT-PCR demonstrated that the eight mRNAs exhibited different expression levels in BC cell lines.

Conclusion: Our study constructed a convenient and reliable 8-mRNA gene signature, which might provide prognostic prediction and aid treatment decision making of $\mathrm{BC}$ patients in clinical practice. 


\section{RESEARCH ARTICLE}

2 Identification and validation of a novel signature for

3 prediction the prognosis and immunotherapy benefit

4 in bladder cancer

5

6

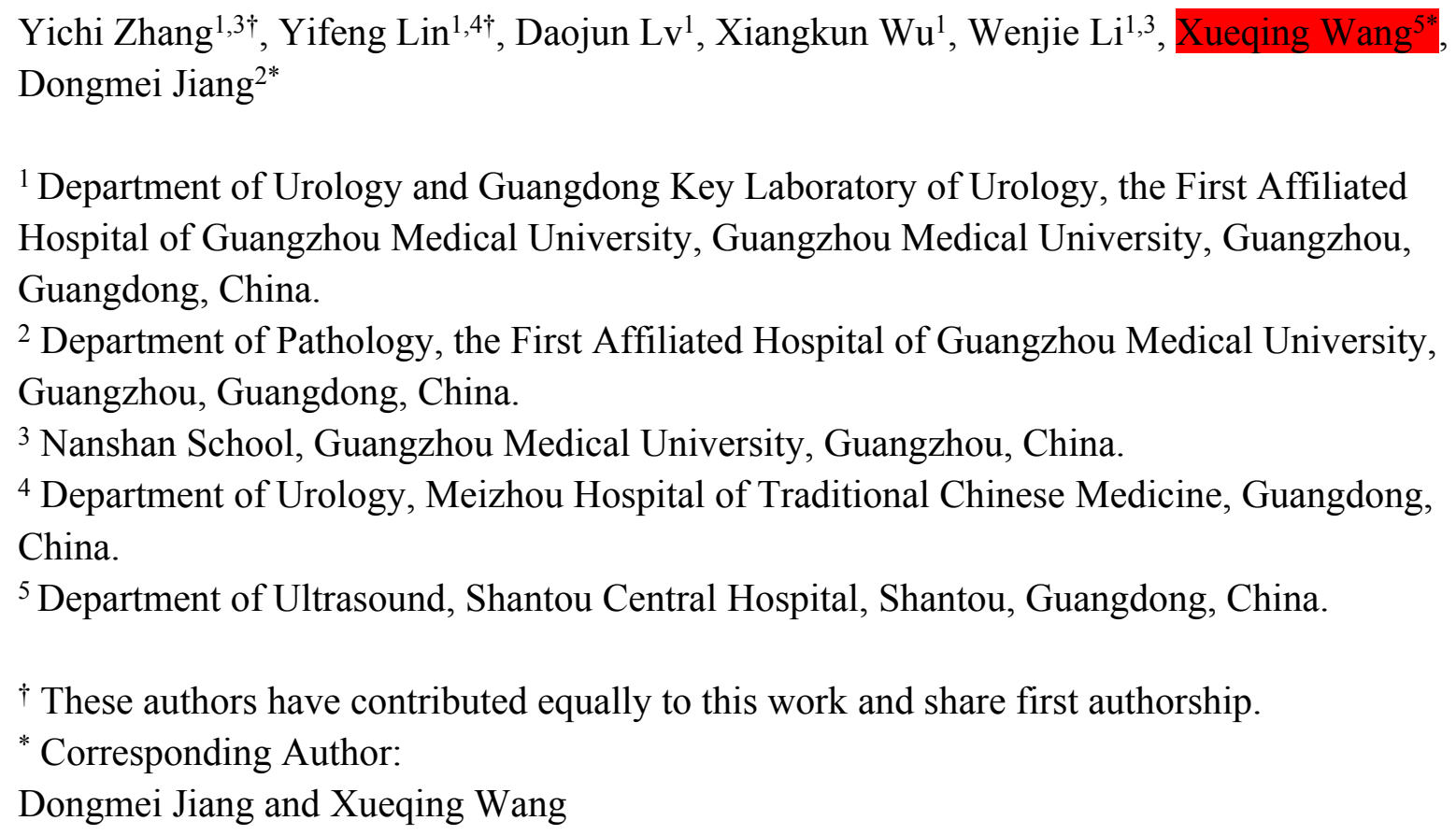

\section{Abstract}

Background: Bladder cancer (BC) is a common urinary tract system tumor with high recurrence rate and different populations show distinct response to immunotherapy. Novel biomarkers that can accurately predict prognosis and therapeutic responses are urgently needed. Here, we aim to identify a novel prognostic and therapeutic responses immune-related gene signature of $\mathrm{BC}$ through a comprehensive bioinformatics analysis.

Methods: The robust rank aggregation was conducted to integrate differently expressed genes (DEGs) in datasets of the Cancer Genome Atlas (TCGA) and the gene expression omnibus (GEO). Lasso and Cox regression analyses were performed to formulate a novel mRNA signature that could predict prognosis of BC patients. Subsequently, the prognostic value and predictive value of the signature was validated with two independent cohorts GSE13507 and IMvigor210. Finally, quantitative Real-time PCR (qRT-PCR) analysis was conducted to determine the expression of mRNAs in BC cell lines (UM-UC-3, EJ-1, SW780 and T24). 
38 Results: We built a signature comprised the eight mRNAs: CNKSR1, COPZ2, CXorf57, FASN, 39 PCOLCE2, RGS1, SPINT1 and TPST1. Our prognostic signature could be used to stratify BC

40 population into two risk groups with distinct immune profile and responsiveness to

41 immunotherapy. The results of qRT-PCR demonstrated that the eight mRNAs exhibited

42 different expression levels in $\mathrm{BC}$ cell lines.

43 Conclusion: Our study constructed a convenient and reliable 8-mRNA gene signature, which 44 might provide prognostic prediction and aid treatment decision making of $\mathrm{BC}$ patients in clinical 45 practice.

46 Keywords: Bladder cancer, Immune checkpoint blockade therapy, Immunogenomic analysis, 47 Tumor microenvironment, Prognosis. 


\section{Introduction}

Bladder cancer (BC) is the most common diagnosed malignancy of the urinary tract system [1]. Each year, bladder cancer is diagnosed in about 430,000 patients and is associated with approximately 165,000 deaths worldwide, making it one of the most lethal cancers [2]. Approximately $75 \%$ of patients with bladder cancers are non-muscle-invasive (NMIBC) at diagnosis and $\sim 25 \%$ are muscle-invasive (MIBC). For BC patients who are at advanced stage (local progression or distant metastasis), cisplatin plus gemcitabine is regarded as the gold standard treatment. However, the anti-tumor effect is not satisfactory due to its low response rate and long-term therapeutic resistance [3]. Currently, with the rapid development of immune checkpoint inhibitors (ICIs) treatment, such as cytotoxic T-lymphocyte antigen 4 (CTLA-4) and programmed cell death molecule $1(P D-1)$ /programmed cell death molecule ligand $1(P D-L 1)$ inhibitors, ICIs are replacing traditional therapeutic drugs and becoming new first-line and second-line treatment options for BC, marking the huge potential and hope of immunotherapy in $\mathrm{BC}$. The median overall survival of $\mathrm{BC}$ patients receiving immunotherapy or chemotherapy was 10.3 months versus 7.4 months [4]. Although ICIs has better efficacy compared with traditional platinum-based chemotherapy, it is estimated that only one fifth of solid tumor patients benefit from the treatment [5]. Moreover, the overall efficacy of ICIs therapies remains unpredictable due to individual heterogeneity of genetics and immune microenvironment alterations as well as multiple confounders (e.g. lifestyle, metabolic disorders and sociological factors) [6-8]. Hence, novel biomarkers that can accurately predict prognosis and therapeutic responses are urgently needed.

Accumulating evidences have confirmed a series of biomarkers including $P D-L 1$ expression, $\mathrm{CD}^{+} \mathrm{T}$ cell, tumor mutational burden (TMB), and microsatellite instability (MSI) could act as biomarkers to predict clinical outcome and therapeutic responses in BC [9-12]. However, these biomarkers seem to be insufficient for the therapeutic options and thus unable to be applied in clinical practice. What's more, incorporating molecular features and clinical information of BC patients into prediction model will provide better prediction effects [13, 14]. Well-validated markers that predict survival benefits and immunotherapy efficacy were still an unmet need in BC.

Owing to advances in high-throughput sequencing, gene signatures at the mRNA level show great potential for predicting patient prognosis. Using transcriptomic profiles from 18 datasets, Aurélie et al. had successfully assigned MIBC patients into six molecular subtypes, in which responses to the treatment regimens may extremely vary [15]. Similarly, in this study we used robust rank aggregation algorithm (RRA) to integrate differently expressed genes in five Gene Expression Omnibus (GEO) datasets. Lasso Cox regression was performed to develop a novel prognostic eight-gene immune signature and the stability and reproducibility was explored in independent datasets. The molecular mechanism and immune landscape relevance of the gene signature and prediction of the potential response to immune checkpoint blockade was investigated. We also validated the expression levels of the eight genes in our clinical samples and cell lines. By applying this gene signature, we could accurately discriminate prognosis in a $\mathrm{BC}$ population and tailored the precision immunotherapy in $\mathrm{BC}$ patients.

\section{Materials \& Methods}




\section{Study design and data collection}

Data were collected as previously described in Zhang et al. (2019) [16]. Specifically, the inclusion criteria of GEO datasets were as follows: (1) biospecimens were gained from patients with localized BC; (2) enrolling at least 5 pairs samples in each dataset; (3) only including transcriptomic data in each dataset. (4) containing both clinical features (clinical tumor stage (TNM) or molecular subtype) and survival outcomes (OS or PFS). The exclusion criteria were as follows: (1) duplicates of the previous eligible datasets; (2) not histo-pathologically confirmed urothelial carcinoma. Gene expression profile data (GSE37815, GSE13507, GSE121711, GSE40355, and GSE3167) were downloaded from the public Gene Expression Omnibus database (GEO, http://www.ncbi.nlm.nih.gov/geo/). Corresponding clinical information for GSE13507 was also obtained. Annotation information for the datasets and the platforms is shown in Table S1. The level 3 RNA-sequencing (RNA-seq) data (Fragments per kilobase million, FPKM) with the corresponding clinical information from 430 BLCA (Bladder Urothelial Carcinoma) samples (19 normal samples and 411 tumor samples) were downloaded from The Cancer Genome Atlas dataset (TCGA) (https://gdc-portal.nci.nih.gov/). The FPKM value was converted to the value per million transcripts (TPM) to make RNA-seq data more comparable with microarray data. The ENSEMBL IDs in RNA-seq data and the probes in microarray data were converted to gene symbol IDs using the annotation files from GENCODE (https ://www.gencodegen es.org/). The IMvigor210 cohort was downloaded from http://researchpub.gene.com/IMvigor210CoreBiologies/\#transcriptome-wide-gene-expression-data. It was a cohort evaluating the effect of atezolizumab ( $P D-L 1$ blockade) in patients with locally advanced or metastatic urothelial BC. A flowchart of the analysis performed in this study is shown in Figure 1.

\section{Data Processing and Screening for Differentially Expressed Genes (DEGs)}

To identify DEGs in the GEO datasets, the raw microarray data was converted to transcripts per million. The robust rank aggregation algorithm (RRA) in the "affy" package was used for background adjustment, $\log 2$ transformation and normalization. Then the RRA method was conducted to integrate the multiple-rank gene list of the five GEO datasets. The "edgeR" package was used to screen the DEGs from the TCGA dataset $(|\log 2 \mathrm{FC}|>1$, adjusted $P<0.05)$. Subsequently, we obtained the intersection of the DEGs from the two datasets by using Venn diagram tool (http://genevenn.sourceforge.net/).

\section{Identification and Estimation of the Prognostic Multi-Gene Signature}

After obtaining the DEGs in the previous step, univariate Cox regression analysis was conducted to determine which gene was significantly correlated with patients' OS $(P<0.05)$. Lassopenalized Cox regression analysis was employed to further minimize the numbers of DEGs. The role of Lasso is to add a constraint condition to the sum of the absolute values of the coefficients, in order to reduce data dimensionality and interference and obtain better fitting. For highthroughput gene expression data with high-dimensional latitude and strong correlations, the Lasso method is practical after compressing meaningless explanatory variables to zero [17]. The regression coefficients of 8 prognosis genes were derived from the stepwise multivariate Cox regression model, and then were used to calculate risk scores. All samples in the training (TCGA-BLCA) sets were divided into high- or low-risk groups by the cutoff values calculated by X-tile software. The Kaplan-Meier survival curves were performed to compare the survival 
133 risk between the two groups. Receiver operating characteristic (ROC) curves were conducted to

134 demonstrate the predictive accuracy of this signature.

135 To determine the predictive power of the prognostic model and other clinical parameters

136 including age, gender, T-stage, $\mathrm{N}$-stage, M-stage, molecular subtype and pathologic stage,

137 univariate and multivariate Cox analyses were performed. The information of six molecular

138 subtypes (luminal papillary, luminal non-specified, luminal unstable, stroma-rich,

$139 \mathrm{basal} / \mathrm{squamous}$, and neuroendocrine-like) were obtained from the supplementary material of the

140 article [15]. Parameters with $P<0.05$ in the univariate analysis were incorporated to conduct the

141 multivariate analysis. Kaplan-Meier analysis was performed to determine whether the effect

142 value was consistent among different subgroups.

\section{Validation of prognostic multi-gene signature}

144 In order to validate the predictive capability of this prognostic model, GSE13507 database was

145 used for external validation. The risk scores were established using the prognostic gene

146 signature. Patients were grouped by the median risk scores using the same method as above.

147 Kaplan-Meier analysis and ROC analysis were generated to evaluate the power of the model.

148 The protein expression levels of the eight genes in normal and BC tissues were validated in the

149 Human Protein Atlas database (https://www.proteinatlas.org/).

\section{Construction and Validation of Predictive Nomogram}

151 The independent clinicopathological parameters identified in multivariate analysis in the previous step were selected to develop a nomogram to predict the 1-, 3-, and 5-year overall survival (OS) of BLCA patients. The AUC of the ROC curve and concordance index were constructed to evaluate the predictive power of this prognostic model, and calibration plot was conducted to compare the accuracy of nomogram-predicted probabilities with actual observation. model.

\section{Functional enrichment analyses}

159 To reveal underlying functions of the prognostic gene signature in $\mathrm{BC}, \mathrm{GO}$ and KEGG analyses 160 were used with a false discovery rate (FDR) $<0.05$ considered statistically significant. Patients

161 from TCGA cohort were classed into two groups based on the risk scores as described

162 previously. GSEA was performed to explore the potential molecular mechanisms enriched in the 163 gene signature (FDR $<5 \%$, nominal $P<1 \%$ and $|\mathrm{NES}|>1$ ).

\section{Tumor Immunity Analyses}

165 To systematically illustrate the relationship between immune infiltrating cell phenotype and BC 166 survival, we applied the CIBERSORT algorithm and assessed the relative proportions of 22

167 distinct leukocyte subsets [18]. Only the CIBERSORT samples with $P<0.05$ were filtered and

168 chosen for further analysis. After filtering the data, the relevant violin plot and correlation

169 heatmap were displayed by R package. The correlation between immune infiltration level and

170 expression of each gene in the gene signature was explored through TIMER2.0

171 (http://timer.comp-genomics.org/). Since the accuracy for estimating the proportion of cell 
172 components were different by applying for different algorithm methods, we used QUANTISEQ

173 algorithm to estimate the changes in the proportion of CD8+ T cell and Tregs, and EPIC

174 algorithm was used to estimate the changes in the proportion of CD4+ T cell, B cell, NK cell,

175 macrophage, cancer associated fibroblast and endothelial cell [19].To further explore the

176 different infiltration degrees of immune cell types, immune-related functions, and immune-

177 related pathways in two risk groups, single sample gene set enrichment analysis (ssGSEA) was

178 applied by using the R package "GSVA" [20]. The correlation between gene signature riskscore

179 and immune related molecules was further investigated to better understand immune infiltration

180 in BLCA.

\section{Prediction and evaluation of immunotherapeutic response}

182 Tumor Immune Dysfunction and Exclusion (TIDE) algorithm were performed to predict the

183 potential response to immune checkpoint blockade. Moreover, an independent cohort

184 (IMvigor210) that recorded expression data from patients who responded or did not respond to

185 anti-PD-L1 immunotherapy was used for further verification. Kaplan-Meier analysis was

186 generated to test the prognostic value of gene signature. CIBERSORT and ssGSEA were applied

187 to explore the immune landscape between high- and low-risk group stratified by risk score.

\section{Cell lines and culture conditions}

189 The human BC cell lines (EJ, T24, UM-UC-3, SW780) and a normal human urinary tract

190 epithelial cell line (SV-HUC-1) were purchased from Stem Cell Bank, Chinese Academy of

191 Sciences (Shanghai, China). SV-HUC-1 was maintained in F-12K medium (Gibco, Grand Island,

192 NY, USA, Cat\#11320033). UM-UC-3, EJ and SW780 cell lines were routinely cultured in RPMI

1931640 medium (Gibco, Cat\#11320033), while T24 cell lines was cultivated in DMEM medium

194 (Gibco, Cat\#11965092). All the media were supplemented with 10\% Fetal Bovine Serum (FBS,

195 Gibco, Cat\#10099141) and 1\% penicillin/streptomycin (Gibco, Cat\#15070063). All cells were

196 incubated at $37{ }^{\circ} \mathrm{C}$ with in an atmosphere of $5 \% \mathrm{CO}_{2}$.

197 Validation of key genes by the quantitative real-time PCR (qRT-PCR) analysis

198 Total RNA was extracted from cultured cell lines using TRIzol reagent (TaKaRa, Dalian, China,

199 Cat\# 9108). The ratio of absorbance at 260 and $280 \mathrm{~nm}$ (the A260/280 ratio) was used to

200 evaluate the purity of RNA. Subsequently, cDNAs were synthesized by using the PrimeScrip ${ }^{\mathrm{TM}}$

201 RT reagent Kit (TaKaRa, Cat\# RR047A) from $1 \mu \mathrm{g}$ of total RNA in $20 \mu 1$ of reaction volume.

$2022 \times \mathrm{TB}^{\circledR}$ Green qPCR Master Mix (TaKaRa, Cat\# RR820Q) was used for qRT-PCR with Roche

203 Lightcycler 480 RT-PCR System. GAPDH fragment was used as an internal control for

204 normalization of the data before calculation using the $2^{-\Delta \Delta \mathrm{Ct}}$ method. Three independent

205 replicates were conducted for each experiment. The primers used are listed in Table S2.

206

207

208

209

210

211

\section{Statistical Analysis}

All statistical analyses were conducted using the R software (version 3.6.2; https://www.rproject.org/). Cox regression model with Lasso based on the R package "glmnet" were performed to generate optimal prognostic signature for BC. The Risk score was calculated using this formula: The risk score $=\sum_{i=1}^{n}(\operatorname{Coefi} *$ Expi $)$, where Expi represents the expression level of gene, $i$ and coefi represents the regression coefficient of gene $i$ in the signature. Kaplan-Meier 
212 plots and log-rank tests were used to compare the difference in the survival status between the

213 high- and low- risk groups. The time-dependent receiver operating characteristic curve (ROC)

214 and calculated the area under the curve (AUC) for 1-year, 3-year, and 5-year OS were conducted

215 to validated the prediction ability of the risk signature. Mann-Whitney-Wilcoxon Test or

216 Student's t test was executed to compare the difference between defined groups for continuous

217 variables. Categorical clinical variables were assessed using Chi-square or Fisher's exact tests.

218 Differences among BC cell lines and urinary tract epithelial cell line were analyzed by one-way

219 ANOVA, and the Dunnett test was used as the post hoc test. $P<0.05$ was set as the cutoffs for

220 statistical significance

\section{Results}

\section{Identification of robust DEGs}

223

224

225

226

227

228

229

230

231

\section{2}

233

234

235

236

237

238

239

240

241

242

243

244

245

246

247

248

249

250

251

The annotation information for the datasets and platforms was exhibited in Table S1. We identified 306, 780, 4035, 452, and 2038 DEGs between normal and tumor tissues in the GEO datasets GSE3167, GSE37815, GSE40355, GSE13507, and GSE121711, respectively. Volcano plots displayed the distribution of DEGs in each dataset (Figures 2A-E). Based on the results of robust rank aggregation method 403 DEGs were screened out, including 132 upregulated and 271 downregulated genes. The top 20 up-regulated and down-regulated DEGs were listed in Figure 2F. A total of 2568 DEGs were selected from the TCGA-BLCA dataset, including 1415 up-regulated and 1153 down-regulated genes. By using Venn diagram web tool we chose a total of 302 DEGs for subsequent analysis in the two cohorts.

\section{Identification and Validation of Eight-Gene Prognostic Signature}

Univariate Cox regression modeling revealed 74-OS related genes in BLCA patients $(P<0.05)$. In the training set (TCGA-BLCA), Lasso-penalized Cox analysis was applied to further analyze the mRNAs data, and 19 genes were identified. The aim of backward stepwise regression was to construct a minimum set of independent variables by including all variables and deleting one variable at a time, to test which was least statistically significant. Model with the highest determination coefficient was built on the remaining variables [21]. 8-prognostic-gene model was finally selected by stepwise regression analysis: CNKSR, COPZ2, CXorf57, FASN, PCOLCE2, RGS1, SPINT1 and TPST1.

The risk scores were calculated for all patient according to the following formula: $(0.0289 \times$ $\left.\exp _{C O P Z 2}\right)-\left(0.0573 \times \exp _{C N K S R I}\right)-\left(0.0602 \times \exp _{C X o r f 57}\right)+\left(0.00678 \times \exp _{F A S N}\right)+(0.0297 \times$ $\left.\exp _{P C O L C E 2}\right)-\left(0.0353 \times \exp _{R G S I}\right)+\left(0.0032 \times \exp _{S P I N T I}\right)+\left(0.04 \times \exp _{\text {TPSTI }}\right)$.

In the training dataset, 394 patients were separated into high- and low-risk groups using the median risk score calculated by $\mathrm{X}$-tile software. The low-risk group showed significant better overall survival compared with the high-risk group $(P<0.001$; Figure 3A). The AUC values of the ROC for 1-, 3-, and 5-year OS were 0.771, 0.735, and 0.718, respectively (Figure 3C). Subgroup analyses stratified by age, gender, AJCC stage and molecular subtype were conducted to evaluate the prognostic values of the eight-gene signature in different subtypes. The patients with high riskscores had worse OS than the patients with low riskscores in age $<65(P<0.0024)$, age $>65(P<0.0001)$, female $(P=0.00011)$, male $(P<0.0001)$, stage III $+\mathrm{IV}(P<0.0001)$,

Peer) reviewing PDF | (2021:09:66073:1:1:NEW 8 Dec 2021) 
252 molecular subtype of basal/squamous $(P=0.0019)$, molecular subtype of luminal papillary $(P=$

253 0.00017) and molecular subtype of luminal non-specified $(P=0.043)$ (Figure S1).

254

255

256

257

258

259

260

261

262

263

264

265

266

267

268

269

270

271

272

273

274

275

276

277

278

279

280

281

282

283

284

285

286

287

288

289

290

\section{Eight-Gene Signature is a Prognostic Factor Independent of Other Clinicopathological Parameters}

In line with the results in the training dataset, the eight-gene signature could successfully stratify the samples in the validation dataset into different risk groups (Figure 3B). The AUC values of the ROC for 1-, 3-, and 5-year OS were 0.677, 0.694, 0.676, respectively (Figure 3D). Cox regression analyses were processed to access whether the eight-gene signature was an independent variable of $\mathrm{BC}$ patient survival. Univariate Cox regression revealed that 8-gene risk score, age, pathologic stage, $\mathrm{T}$ stage, and $\mathrm{N}$ stage were correlated with OS in BC (Figure 3E). After the multivariate Cox analysis, risk score, age, and pathologic stage remained as independent prognostic parameters (Figure 3F). The expression of CNKSR1, CXorf57 and FASN at the protein level were significantly elevated in $\mathrm{BC}$ tissues compared with non-cancerous tissues, while the level of COPZ2, PCOLCE2, TPST1 and RGS1 were notably decreased in BC tissues than in normal tissues. No difference was found for SPINT1 protein expression (Figure S2). In addition, high risk group was correlated with a higher histological grade, $\mathrm{T}$ stage, $\mathrm{N}$ stage, M stage and clinical stage as shown in Table 1.

\section{Construction and Validation of the Predictive Nomogram}

In order to predict the prognosis of BLCA patients, we integrated the independent prognostic parameters, including 8-gene risk score, age and AJCC stage, to develop a nomogram model. (Figure 4A). The AUCs for the 1-, 3-, and 5-year OS were 0.769, 0.751, and 0.764, respectively (Figure 4B). The C-index of the nomogram model was 0.703 (95\% CI; 0.547-0.872), whereas that for AJCC stage was 0.634 (95\% CI; 0.451-0.818), with 1000 cycles of bootstrapping. Calibration plots showed that the results of predicted OS were consistent with the actual observations. (Figure 4C). DCA was used to evaluate the clinical utility of the nomogram model. The nomogram showed the greatest net benefit when compared with AJCC stage, T stage and risk score alone. (Figure 4D).

\section{GO/KEGG/GSEA}

In biological processes, the gene signature was significantly enriched in focal adhesion, microRNAs in cancer, proteoglycans in cancer, cell cycle, and extracellular matrix receptor interaction; these processes have close connections with tumor proliferation and metastasis (Figure 5A). The KEGG pathway analysis gave similar results (Figure 5B).

To explore the significantly enriched pathways of the eight prognostic genes GSEA was performed. The results showed the high-risk group was enriched in more T cell suppressive pathways, such as the transforming growth factor $\beta$ (TGF- $\beta$ ) signaling pathway, Wnt signaling pathway, and calcium signaling pathway (Figure 5C).

\section{Correlation between tumor immunity and eight-gene signature}

The association between risk score and the distribution of tumor-infiltrating immune cells was further investigated in two risk groups. Results showed that significantly higher proportions of

Peer) reviewing PDF | (2021:09:66073:1:1:NEW 8 Dec 2021) 
291 M0 macrophages $(P=0.013)$, M2 macrophage $(P=0.031)$ and resting mast cells $(P=0.04)$

292 were exhibited in the high-risk group while the proportions of CD8 ${ }^{+} \mathrm{T}$ cells $(P<0.001), \mathrm{CD} 4$

293 memory-activated T cells $(P=0.001)$, regulatory T cells (Tregs) $(P=0.003)$ and follicular

294 helper T cells $(P=0.034)$ were dramatically lower in the high-risk group (Figure 6A-B). It's

295 worth noting that the expression levels of eight genes were positively correlated with

296

297 macrophage and cancer associated fibroblast infiltration (Figure S3). BLCA samples were successfully divided into two clusters by applying "GSVA" algorithm. The ESTIMATE Score,

298

299 Immune Score and Stromal Score were significantly higher in low-risk group than that of highrisk group. Also, low risk group had higher infiltration degrees of immune-related functions as

300

301

302 well as immune-related pathways compared with high- risk group (Figure 6C). Interestingly, the risk score was strongly positively correlated with the expression of immune checkpoints such as $P D-1, P D-L 1, C T L A-4, T I M 3, L A G 3$ and TIGIT (Figure 6D).

303

304

305

306

307

308

309

310

311

312

313

314

315

316

317

318

319

320

321

322

323

324

325

326

327

328

329

\section{Verification of the Correlation Between the Risk Signature and Immunotherapeutic response}

The CTLA-4 and $P D-1 / P D-L 1$ blockade has emerged as a critical weapon in treating cancer [22]. Thus, we evaluated the eight-gene signature in predicting the sensitivity to ICBs in the two groups through TIDE algorithm and subclass mapping. The results showed that BLCA patients in low-risk group were more likely to response to $P D-1$ blockade (Bonferroni corrected $P=$ 0.014), and high-risk groups were more likely to response to CTLA-4 blockade (Bonferroni corrected $P=0.041$ ) (Figure 7A). We investigated the prognostic value of the predictive model in IMvigor 210 cohort, which included patients with metastatic urothelial cancer treated with anti-PD-L1 antibody. The results showed low-risk group stratified by the eight gene signature has a significant survival benefit compared with high-risk group (Figure 7B). The validation cohort also showed a similar immune pattern with training cohort, and the risk score was found to be significantly correlated with immune-related molecules (Figure 7C-F).

\section{Distribution of risk score in different $\mathrm{BC}$ molecular subtypes}

According to different transcriptomic and genomic profiling $\mathrm{BC}$ can be classified into six molecular subtypes, each with different clinical prognosis and therapeutic responses to chemotherapy and immunotherapy. We evaluated the distribution of risk score in six BC molecular subtypes, and the results showed the risk score was significantly different in different molecular subtypes (Figure 8). Ba/Sq tumor with poor prognosis had the highest risk score, but the best prognostic outcome LumP and LumU had the lowest risk score.

\section{Validation of the expression levels of the 8 genes in cell lines}

The expression signatures at the mRNA level of the 8 genes were then investigated in four human BC cell lines (i. e. EJ, T24, UM-UC-3 and SW780) and demonstrated that the mRNA levels of CNKSRl were relatively higher in most of the BC cells when compared with normal human urinary tract epithelial cell line (SV-HUC-1), however CXorf57 and FASN were dramatically decreased in most of the BC cells (Figure S4 A-H).

\section{Discussion}


330 Considerable heterogeneity at the genomic, transcriptional, and cellular levels have been found 331 in BC, which may result in diverse clinical characteristics and contribute to different responses to 332 chemotherapy, immunotherapy, and targeted therapies [23]. Genetic biomarkers have identifiable

333

334

335

336

337

338

339

340

341

342

343

344

345

346

347

348

349

350

351

352

353

354

355

356

357

358

359

360

361

362

363

364

365

366

367

368

369

370

371

372

373

374 molecular characteristics that can be used to detect disease, evaluate prognosis, and monitor tumor progression or therapeutic response [24]. Previous reports have demonstrated promising gene-based risk signature models that enable immune monitoring of tumors and individualized treatment [25-27]. As a new treatment method, ICIs exert a favorable effect in patients with BC. However, some patients show unresponsiveness to ICIs treatment. We hope to identify a prognostic gene signature which can not only predict the prognosis of $\mathrm{BC}$ patients but also select the potential patients that may show favorable response to ICIs.

In this study, a panel of eight-gene signature comprising CNKSR1, CXorf57, FASN, SPINT1, COPZ2, PCOLCE2, TPST1, and RGS1 was finally selected to generate a risk score model which can be exploited for predicting survival in $\mathrm{BC}$. The predictive performance of the signature was mutually verified in internal TCGA-BLCA and external GSE13507 dataset. Survival analysis revealed that high-risk group exhibited significantly worse prognosis than low-risk group. A nomogram integrated with both the 8-gene-based signature and clinicopathological risk factors demonstrated that the model can accurately predict patients' overall survival (OS). The AUC, Cindex, DCA, and calibration curves all indicated the good predictive performance of this model. The risk score showed a powerful ability to differentiate BLCA into different risk groups and, together with the nomogram, could facilitate the clinical use in clinical practice.

Interestingly, these eight genes which were used to establish our risk signature model have been shown to be involved in tumor development and thus may be promising therapeutic targets for BC. FASN has previously been reported as an oncogene in many cancer types [28]. Its product is the only human protein to catalyze de novo synthesized long-chain fatty acids, implying FASN plays a crucial role in lipid metabolism in tumor microenvironment. The upregulation of FASN enhances lipogenesis in tumor cells, mainly through activation of the AKT/mTOR signaling pathway, thereby contributing to the immunometabolic switch in the tumor microenvironment and enhancing tumor growth and proliferation. In addition, metformin, a commonly used drug for treating diabetes, has exerted potent inhibitory effect in tumor growth through targeting SREBP-1c and its downstream target FASN, thus inhibiting lipogenesis in bladder cancer [29]. Studies have shown that blockade of FASN exerts a novel effect in inhibiting growth of BC cells [30, 31]. SPINT1 (HAI-1, hepatocyte growth factor activator inhibitor type 1), is an inhibitor of transmembrane serine protease that regulates matriptase activity. Hepatocyte growth factor $(H G F)$ contribute to tumor development through its specific receptor MET [32, 33]. Most fibroblasts secrete the inactive single-chain precursor (pro- $H G F$ ) and matriptase is one of the most potent activators of pro- $H G F$ [34]. HAI-1 suppresses matriptase-mediated conversion of pro- $H G F$ into its active form [35]. Shimwell et al. in their study found a significantly elevated concentration of $H A I-1$ in the urine sample and identified HAI-1 as potential urinary biomarker through the combination of transcriptomics and proteomic analyses [36]. HAI-1 may also be involved in cell migration and bladder cancer cell metastasis [37]. Yamasaki et al. revealed that low expression of HAI-1 was related to poor prognosis in BC [38]. However, this was inconsistent with our results. Further experiments are needed to elucidate the mechanism by which HAI-1 participates in the development of BC. CXorf57, also known as RADX, is a singlestranded DNA-binding protein. By modulating the activity of RAD51, which is known to be involved in the homologous recombination and repair of DNA, it promotes replication fork

Peer) reviewing PDF | (2021:09:66073:1:1:NEW 8 Dec 2021) 
375 stability $[39,40]$. CNKSRI acts as a scaffold component for receptor tyrosine kinase signaling

376 and is involved in many signal transduction pathways, including PI3K-Akt, Ras, MAPK, and NF-

$377 \kappa B$ signaling [41-45]. Overexpression of CNKSR1 may promote proliferation and invasion in

378 human breast and cervical cancer [41, 42]. However, other studies have shown that high

379 CNKSR1 expression was associated with less aggressive biological characteristics of pancreatic

380 cancer, suggesting that it may be helpful in the selection of patients for surgical resection [46].

381 Wang et al. found that CNKSR1 protein was mainly expressed in the cytoplasm and the

382 expression of CNKSR1 protein was significantly higher in MIBC than in normal bladder

383 samples [47]. However, its underlying role in remaining bladder tumor growth remained unclear.

$384 C O P Z 1$ and COPZ2 are two isoforms encoding the $\zeta$ subunit of coat protein complex 1, which functions as a vesicle carrier in secretory pathways [48]. Downregulation of miR-152 and its host gene COPZ2, together with upregulation of COPZ1 led to suppression of autophagy and apoptosis in cancer cells $[49,50]$. PCOLCE2 encodes a glycoprotein, procollagen $\mathrm{COOH}-$ terminal proteinase enhancer $(P C P E)$, which regulates collagen fibril deposition in the extracellular matrix and plays an important role in remodeling the tumor microenvironment [51]. PCOLCE2 is regarded as a biomarker for gynecological cancers, squamous cell carcinoma of head and neck and non-small-cell lung cancer [52-54]. Consistent with our results, low PCOLCE2 expression is linked to better OS. Tyrosine sulfation, which is catalyzed by tyrosyl protein sulfotransferase 1 (TPST1) and TPST2, leads to changes in protein-tyrosine sulfotransferase, transferase, and protein homodimerization activity. TPST1 is overexpressed in nasopharyngeal carcinoma and is associated with tumor invasion [55]. Expression levels of TPST1 in lung cancer tissue are significantly lower than those in normal tissue and are inversely association with the expression of $c-$ Met [56]. However, the biological function of TPST1 in BC remains unclear. $R G S 1$ is a GTPase-activating protein and it can regulate the function of Gproteins [57]. Studies have shown RGS1 can desensitize chemokine receptor signaling and cause decreased chemotaxis of lymphocytes in lymphoid organs [58]. We imputed RGSI may lead to lymphocyte dysfunction and contribute to immune escape in BC.

To uncover the molecular mechanisms of the gene signature and their potential biological functions, GO, KEGG, and GSEA enrichment analyses were executed. Based on the results of GO and KEGG analysis, we found that the eight genes may play important parts in tumor proliferation and metastasis. GSEA revealed enrichment of $T G F-\beta$ and $\mathrm{Wnt} / \beta$-catenin signaling in the high-risk group. $T G F-\beta$ is an immunosuppressive factor that plays a crucial part in cancer development through promoting dysplasia, angiogenesis and epithelial-to-mesenchymal transition. Also, TGF- $\beta$ may impair the anti-tumor T cell response [59]. Mariathasan et al. revealed that blockade of $T G F-\beta$ enhanced the potency of anti- $P D-L 1$ antibody (atezolizumab) by boosting the activity of $\mathrm{CD} 8^{+} \mathrm{T}$ cells and hence enhancing anti-tumor immunity [60]. In urothelial cancers key molecules that regulate the Wnt/ $\beta$-catenin pathways are being used as diagnostic biomarkers and potential therapeutic targets [61]. Spranger et al. found that aberrant Wnt $\beta$-catenin activation led to defects in $\mathrm{CD} 103^{+}$dendritic cells recruitment and a subsequent decrease in $C X C L 9$ and $C X C L 10$ secretion, thereby preventing T-cell infiltration [62]. We also evaluated the correlation between tumor immunity and the eight gene signature both in internal TCGA-BLCA and external IMvigor210 cohort. Our results revealed that significantly higher levels of $\mathrm{CD}^{+} \mathrm{T}$ cells, $\mathrm{CD} 4^{+} \mathrm{T}$ cells and lower levels of macrophages were exhibited in low-risk group than in high-risk group. The expression levels of eight genes were positively correlated with macrophages and cancer associated fibroblast infiltration, and these cells played an important role in the creation of immunosuppressive TME and had a negative prognostic effect 
421 on BC. In addition, risk score was also positively correlated with immune checkpoint molecules

422

423

424

425

426

427

428

429

430

431

432

433

434

435

436

437

438

439

440

441

442

443

444

445

446

447

448

449

450

451

452

453

454

455

456

457

458

459

\section{Conclusions}

461

462

463

such as $P D-1, P D-L 1$ and $C T L A 4$. We imputed that once these immune checkpoint molecules were activated, they may promote tumor cells and other "bad cells" to escape the immune system. The landscapes of immune infiltrating cells, immune-related function and immunerelated pathways of the high- and low-risk groups indicated that the low-risk group tended to have inflamed tumors, whereas the high-risk group had excluded tumors [63]. Increasing evidences have indicated that immune inflamed subtypes which characterized by infiltration of $\mathrm{CD} 8^{+} \mathrm{T}$ cells have optimal response to ICIs therapy [63-65]. Excluded tumors are a subtype characterized by retention of cytotoxic $\mathrm{T}$ cell in excessive reactive stroma but not the absence of $\mathrm{T}$ cells. Thus, this kind of tumor is still responsive to immune checkpoint inhibitors.

TIDE algorithm indicated that low-risk BLCA patients may be responsive to $P D-1$ blockade therapy, and high-risk BLCA patients may be responsive to CTLA4 blockade therapy. Based on the above results we imputed that ICIs may still yield survival benefits in high-risk BLCA patients but the normal anti-tumor immunity needed to be restored within tumor parenchyma (such as inhibition of fibroblasts in tumor stroma, elimination or transformation of tumorassociated macrophages (TAMs), Tregs and myeloid-derived suppressor cells (MDSCs), inhibition of TGF- $\beta$ and Wnt/ $\beta$-catenin signaling. Recent research on neutralization of TGF- $\beta$ led to tumor stroma remodeling and enhanced the efficacy of immunotherapy also provide rationale for "targeted + immune" treatment in immune excluded tumors [66]. These results showed great effectiveness of our eight-gene-based risk signature in distributing BC patients into inflamed or excluded subtype, which may benefit from different ICIs based treatment. It might serve as a biomarker in tailoring individualized immunotherapy.

To our knowledge, the eight-gene signature presented here has not been previously reported and is more cost-effective and practical in clinical utility than whole-genome sequencing. Considering the intra- and inter-tumor heterogeneity of $\mathrm{BC}$ tumors we sought to find variable in transcriptional levels which are more suitable to explain the complex interplay between tumors and the immune system, when compared to models incorporated clinical parameters only. We used rank aggregation analysis and integrated five GEO and TCGA datasets to identify the final gene signature. Hence, our results are highly reliable and robust. As BC is a heterogeneous cancer and can be classified into different molecular subtypes, each with different clinical prognosis and therapeutic responses to chemotherapy and immunotherapy; and we found risk score was significantly different in different molecular subtypes. The results of RNA-sequence in cell lines demonstrated that aberrant expression levels of CNKSR1, CXorf57 and FASN may promote proliferation and induce malignant transformation in BC. Moreover, a nomogram combining the eight-gene signature with clinicopathological parameters further improved the predictive power and the possibility of clinical use. Although the risk signature exerts a robust predictive value in risk stratification and guidance for treatment options, its accuracy and effectiveness need to be further confirmed in substantial clinical trials. Also, in vivo studies investigating the role of the eight genes are needed to verify our results in the future.

In summary, our study identified a novel signature that would be applied as a prognosticator and a promising biomarker in individualized immunotherapy for BC. These findings improve our understanding of immunotherapies in $\mathrm{BC}$ and provide valuable indication for future studies.

Peer) reviewing PDF | (2021:09:66073:1:1:NEW 8 Dec 2021) 


\section{Abbreviations}

465 BC bladder cancer; ICIs immune checkpoint inhibitors; TME tumor microenvironment; OS 466 overall survival; HR hazard ratio; CI confidence interval; AJCC American Joint Committee on 467 Cancer; DEGs differentially expressed genes; AUC Area Under the Curve; ROC Receiver468 operator Characteristic curve; Lasso least absolute shrinkage and selection operator; GSEA gene 469 set enrichment analysis; GO gene ontology; KEGG Kyoto Encyclopedia of Genes and 470 Genomes; TCGA-BLCA The Cancer Genome Atlas Bladder Urothelial Carcinoma; GEO Gene 471 Expression Omnibus.

\section{Acknowledgements}

473 Not applicable.

\section{References}

475 1. Torre LA, Bray F, Siegel RL, Ferlay J, Lortet-Tieulent J, Jemal A. Global cancer statistics, 476 2012. CA: a cancer journal for clinicians. 2015;65(2):87-108. 477 http://dx.doi.org/10.3322/caac.21262

478 2. Kamat AM, Hahn NM, Efstathiou JA, Lerner SP, Malmström PU, Choi W, et al. Bladder 479 cancer. Lancet (London, England). 2016;388(10061):2796-810. http://dx.doi.org/10.1016/s0140$480 \quad \frac{6736(16) 30512-8}{3 .}$

481 3. Kaufman D, Raghavan D, Carducci M, Levine EG, Murphy B, Aisner J, et al. Phase II trial 482 of gemcitabine plus cisplatin in patients with metastatic urothelial cancer. Journal of clinical 483 oncology : official journal of the American Society of Clinical Oncology. 2000;18(9):1921-7. 484 http://dx.doi.org/10.1200/jco.2000.18.9.1921

485 4. Bellmunt J, de Wit R, Vaughn DJ, Fradet Y, Lee JL, Fong L, et al. Pembrolizumab as 486 Second-Line Therapy for Advanced Urothelial Carcinoma. The New England journal of medicine. 487 2017;376(11):1015-26. http://dx.doi.org/10.1056/NEJMoa1613683

488 5. Fares CM, Van Allen EM, Drake CG, Allison JP, Hu-Lieskovan S. Mechanisms of 489 Resistance to Immune Checkpoint Blockade: Why Does Checkpoint Inhibitor Immunotherapy Not 490 Work for All Patients? American Society of Clinical Oncology educational book American 491 Society of Clinical Oncology Annual Meeting. 2019;39:147-64. 492 http://dx.doi.org/10.1200/edbk 240837

4936 Deshpande RP, Sharma S, Watabe K. The Confounders of Cancer Immunotherapy: Roles 494 of Lifestyle, Metabolic Disorders and Sociological Factors. Cancers. 2020;12(10). 495 http://dx.doi.org/10.3390/cancers12102983

$4967 . \quad$ Desrichard A, Kuo F, Chowell D, Lee KW, Riaz N, Wong RJ, et al. Tobacco Smoking497 Associated Alterations in the Immune Microenvironment of Squamous Cell Carcinomas. Journal 498 of the National Cancer Institute. 2018;110(12):1386-92. http://dx.doi.org/10.1093/jnci/djy060

499 8. Barr T, Girke T, Sureshchandra S, Nguyen C, Grant K, Messaoudi I. Alcohol Consumption 500 Modulates Host Defense in Rhesus Macaques by Altering Gene Expression in Circulating 501 Leukocytes. Journal of immunology (Baltimore, Md : 1950). 2016;196(1):182-95. 502 http://dx.doi.org/10.4049/jimmunol.1501527

503 9. Patel SP, Kurzrock R. PD-L1 Expression as a Predictive Biomarker in Cancer 504 Immunotherapy. Molecular cancer therapeutics. 2015;14(4):847-56.

505 http://dx.doi.org/10.1158/1535-7163.Mct-14-0983 
506 10. Chan TA, Yarchoan M, Jaffee E, Swanton C, Quezada SA, Stenzinger A, et al. 507 Development of tumor mutation burden as an immunotherapy biomarker: utility for the oncology 508 clinic. Annals of oncology : official journal of the European Society for Medical Oncology. 2019;30(1):44-56. http://dx.doi.org/10.1093/annonc/mdy495

510 11. Liu Z, Zhou Q, Wang Z, Zhang H, Zeng H, Huang Q, et al. Intratumoral TIGIT(+) CD8(+)

511 T-cell infiltration determines poor prognosis and immune evasion in patients with muscle-invasive

512 bladder cancer. Journal for immunotherapy of cancer. 2020;8(2). http://dx.doi.org/10.1136/jitc-

$513 \quad 2020-000978$

514 12. Necchi A, Madison R, Raggi D, Jacob JM, Bratslavsky G, Shapiro O, et al. Comprehensive

515 Assessment of Immuno-oncology Biomarkers in Adenocarcinoma, Urothelial Carcinoma, and

516 Squamous-cell Carcinoma of the Bladder. European urology. 2020;77(4):548-56.

517 http://dx.doi.org/10.1016/j.eururo.2020.01.003

518 13. Kim WJ, Kim SK, Jeong P, Yun SJ, Cho IC, Kim IY, et al. A four-gene signature predicts 519 disease progression in muscle invasive bladder cancer. Molecular medicine (Cambridge, Mass). 520 2011;17(5-6):478-85. http://dx.doi.org/10.2119/molmed.2010.00274

521 14. Song BN, Kim SK, Mun JY, Choi YD, Leem SH, Chu IS. Identification of an 522 immunotherapy-responsive molecular subtype of bladder cancer. EBioMedicine. 2019;50:238-45. http://dx.doi.org/10.1016/j.ebiom.2019.10.058

15. Kamoun A, de Reyniès A, Allory Y, Sjödahl G, Robertson AG, Seiler R, et al. A Consensus Molecular Classification of Muscle-invasive Bladder Cancer. European urology. 2020;77(4):42033. http://dx.doi.org/10.1016/j.eururo.2019.09.006

16. Zhang Q, Xia J, Wang Y, Zhang J, Ji C, Cong R, et al. Tumor infiltrating M2 macrophages could predict biochemical recurrence of localized prostate cancer after radical prostatectomy. Experimental cell research. 2019;384(1):111588. http://dx.doi.org/10.1016/j.yexcr.2019.111588

530 17. Gui J, Li H. Penalized Cox regression analysis in the high-dimensional and low-sample 531 size settings, with applications to microarray gene expression data. Bioinformatics (Oxford, 532 England). 2005;21(13):3001-8. http://dx.doi.org/10.1093/bioinformatics/bti422

533 18. Newman AM, Liu CL, Green MR, Gentles AJ, Feng W, Xu Y, et al. Robust enumeration 534 of cell subsets from tissue expression profiles. Nature methods. 2015;12(5):453-7. 535 http://dx.doi.org/10.1038/nmeth.3337

536 19. Sturm G, Finotello F, Petitprez F, Zhang JD, Baumbach J, Fridman WH, et al. 537 Comprehensive evaluation of transcriptome-based cell-type quantification methods for immuno538 oncology. Bioinformatics (Oxford, England). 2019;35(14):i436-i45. 539 http://dx.doi.org/10.1093/bioinformatics/btz363

540 20. Hänzelmann S, Castelo R, Guinney J. GSVA: gene set variation analysis for microarray 541 and RNA-seq data. BMC bioinformatics. 2013;14:7. http://dx.doi.org/10.1186/1471-2105-14-7

542 21. Kuhn M, Johnson K. Applied predictive modeling: Springer; 2013.

543 22. Postow MA, Callahan MK, Wolchok JD. Immune Checkpoint Blockade in Cancer 544 Therapy. Journal of clinical oncology : official journal of the American Society of Clinical 545 Oncology. 2015;33(17):1974-82. http://dx.doi.org/10.1200/jco.2014.59.4358

546 23. Meeks JJ, Al-Ahmadie H, Faltas BM, Taylor JA, 3rd, Flaig TW, DeGraff DJ, et al. 547 Genomic heterogeneity in bladder cancer: challenges and possible solutions to improve outcomes. 548 Nature reviews Urology. 2020;17(5):259-70. http://dx.doi.org/10.1038/s41585-020-0304-1

549 24. Khailany RA, Aziz SA, Najjar SM, Safdar M, Ozaslan M. Genetic biomarkers: Potential 550 roles in cancer diagnosis. Cellular and molecular biology (Noisy-le-Grand, France). 2020;66(3):15517. 
552 25. Qu L, Wang ZL, Chen Q, Li YM, He HW, Hsieh JJ, et al. Prognostic Value of a Long Non553 coding RNA Signature in Localized Clear Cell Renal Cell Carcinoma. European urology. 554 2018;74(6):756-63. http://dx.doi.org/10.1016/j.eururo.2018.07.032

555 26. Lu H, Liang Y, Guan B, Shi Y, Gong Y, Li J, et al. Aristolochic acid mutational signature 556 defines the low-risk subtype in upper tract urothelial carcinoma. Theranostics. 2020;10(10):4323557 33. http://dx.doi.org/10.7150/thno.43251

558 27. Wang S, Zhang Q, Yu C, Cao Y, Zuo Y, Yang L. Immune cell infiltration-based signature 559 for prognosis and immunogenomic analysis in breast cancer. Briefings in bioinformatics.

560

561

562

563

564

565

566

567

568

569

570

571

572

573

574

575

576

577 2021;22(2):2020-31. http://dx.doi.org/10.1093/bib/bbaa026

28. Menendez JA, Lupu R. Fatty acid synthase and the lipogenic phenotype in cancer pathogenesis. Nature reviews Cancer. 2007;7(10):763-77. http://dx.doi.org/10.1038/nrc2222

29. Deng J, Peng M, Zhou S, Xiao D, Hu X, Xu S, et al. Metformin targets Clusterin to control lipogenesis and inhibit the growth of bladder cancer cells through SREBP-1c/FASN axis. Signal transduction and targeted therapy. 2021;6(1):98. http://dx.doi.org/10.1038/s41392-021-00493-8

30. Tao T, Su Q, Xu S, Deng J, Zhou S, Zhuang Y, et al. Down-regulation of PKM2 decreases FASN expression in bladder cancer cells through AKT/mTOR/SREBP-1c axis. Journal of cellular physiology. 2019;234(3):3088-104. http://dx.doi.org/10.1002/jcp.27129

31. Zheng SS, Gao JG, Liu ZJ, Zhang XH, Wu S, Weng BW, et al. Downregulation of fatty acid synthase complex suppresses cell migration by targeting phosphor-AKT in bladder cancer. Molecular medicine reports. 2016;13(2):1845-50. http://dx.doi.org/10.3892/mmr.2015.4746

32. Tervonen TA, Belitškin D, Pant SM, Englund JI, Marques E, Ala-Hongisto H, et al. Deregulated hepsin protease activity confers oncogenicity by concomitantly augmenting HGF/MET signalling and disrupting epithelial cohesion. Oncogene. 2016;35(14):1832-46. http://dx.doi.org/10.1038/onc.2015.248

33. Kawaguchi M, Kataoka H. Mechanisms of hepatocyte growth factor activation in cancer tissues. Cancers. 2014;6(4):1890-904. http://dx.doi.org/10.3390/cancers6041890

578 34. Lee SL, Dickson RB, Lin CY. Activation of hepatocyte growth factor and 579 urokinase/plasminogen activator by matriptase, an epithelial membrane serine protease. The

580 Journal of biological chemistry. $2000 ; 275(47): 36720-5$. http://dx.doi.org/10.1074/jbc.M007802200

582 35. Ye F, Chen S, Liu X, Ye X, Wang K, Zeng Z, et al. 3-Cl-AHPC inhibits pro-HGF 583 maturation by inducing matriptase/HAI-1 complex formation. Journal of cellular and molecular 584 medicine. 2019;23(1):155-66. http://dx.doi.org/10.1111/jcmm.13900

36. Shimwell NJ, Bryan RT, Wei W, James ND, Cheng KK, Zeegers MP, et al. Combined proteome and transcriptome analyses for the discovery of urinary biomarkers for urothelial carcinoma. British journal of cancer. 2013;108(9):1854-61. http://dx.doi.org/10.1038/bjc.2013.157

589 37. Chen Y, Xu T, Xie F, Wang L, Liang Z, Li D, et al. Evaluating the biological functions of 590 the prognostic genes identified by the Pathology Atlas in bladder cancer. Oncology reports. 591 2021;45(1):191-201. http://dx.doi.org/10.3892/or.2020.7853

592 38. Yamasaki K, Mukai S, Nagai T, Nakahara K, Fujii M, Terada N, et al. Matriptase-Induced 593 Phosphorylation of MET is Significantly Associated with Poor Prognosis in Invasive Bladder 594 Cancer; an Immunohistochemical Analysis. International journal of molecular sciences. 595 2018;19(12). http://dx.doi.org/10.3390/ijms19123708

597 single-stranded DNA to promote replication fork stability. EMBO reports. 2017;18(11):1991- 
598

599

600

601

602

603

604

605

606

607

608

609

610

611

612

613

614

615

616

617

618

619

620

621

622

623

624

625

626

627

628

629

630

631

632

633

634

635

636

637

638

639

640

641

642

643

2003. http://dx.doi.org/10.15252/embr.201744877

40. Dungrawala H, Bhat KP, Le Meur R, Chazin WJ, Ding X, Sharan SK, et al. RADX Promotes Genome Stability and Modulates Chemosensitivity by Regulating RAD51 at Replication Forks. Molecular cell. 2017;67(3):374-86.e5. http://dx.doi.org/10.1016/j.molcel.2017.06.023

41. Fritz RD, Varga Z, Radziwill G. CNK1 is a novel Akt interaction partner that promotes cell proliferation through the Akt-FoxO signalling axis. Oncogene. 2010;29(24):3575-82. http://dx.doi.org/10.1038/onc.2010.104

42. Fritz RD, Radziwill G. CNK1 promotes invasion of cancer cells through NF-kappaBdependent signaling. Molecular cancer research : MCR. 2010;8(3):395-406. http://dx.doi.org/10.1158/1541-7786.Mcr-09-0296

43. Indarte M, Puentes R, Maruggi M, Ihle NT, Grandjean G, Scott M, et al. An Inhibitor of the Pleckstrin Homology Domain of CNK1 Selectively Blocks the Growth of Mutant KRAS Cells and Tumors. Cancer research. 2019;79(12):3100-11. http://dx.doi.org/10.1158/0008-5472.Can$18-2372$

44. Farhan H, Wendeler MW, Mitrovic S, Fava E, Silberberg Y, Sharan R, et al. MAPK signaling to the early secretory pathway revealed by kinase/phosphatase functional screening. The Journal of cell biology. 2010;189(6):997-1011. http://dx.doi.org/10.1083/jcb.200912082

45. Fischer A, Warscheid B, Weber W, Radziwill G. Optogenetic clustering of CNK1 reveals mechanistic insights in RAF and AKT signalling controlling cell fate decisions. Scientific reports. 2016;6:38155. http://dx.doi.org/10.1038/srep38155

46. Quadri HS, Aiken TJ, Allgaeuer M, Moravec R, Altekruse S, Hussain SP, et al. Expression of the scaffold connector enhancer of kinase suppressor of Ras 1 (CNKSR1) is correlated with clinical outcome in pancreatic cancer. BMC cancer. 2017;17(1):495. http://dx.doi.org/10.1186/s12885-017-3481-4

47. Wang L, Liu X, Yue M, Liu Z, Zhang Y, Ma Y, et al. Identification of hub genes in bladder cancer based on weighted gene co-expression network analysis from TCGA database. Cancer reports (Hoboken, NJ). 2021:e1557. http://dx.doi.org/10.1002/cnr2.1557

48. Beck R, Rawet M, Wieland FT, Cassel D. The COPI system: molecular mechanisms and function. FEBS letters. 2009;583(17):2701-9. http://dx.doi.org/10.1016/j.febslet.2009.07.032

49. Shtutman M, Baig M, Levina E, Hurteau G, Lim CU, Broude E, et al. Tumor-specific silencing of COPZ2 gene encoding coatomer protein complex subunit $\zeta 2$ renders tumor cells dependent on its paralogous gene COPZ1. Proceedings of the National Academy of Sciences of the United States of America. 2011;108(30):12449-54. http://dx.doi.org/10.1073/pnas.1103842108

50. Tsuruta T, Kozaki K, Uesugi A, Furuta M, Hirasawa A, Imoto I, et al. miR-152 is a tumor suppressor microRNA that is silenced by DNA hypermethylation in endometrial cancer. Cancer research. 2011;71(20):6450-62. http://dx.doi.org/10.1158/0008-5472.Can-11-0364

51. Steiglitz BM, Keene DR, Greenspan DS. PCOLCE2 encodes a functional procollagen Cproteinase enhancer (PCPE2) that is a collagen-binding protein differing in distribution of expression and post-translational modification from the previously described PCPE1. The Journal of biological chemistry. 2002;277(51):49820-30. http://dx.doi.org/10.1074/jbc.M209891200

52. Tian S, Meng G, Zhang W. A six-mRNA prognostic model to predict survival in head and neck squamous cell carcinoma. Cancer management and research. 2019;11:131-42. http://dx.doi.org/10.2147/cmar.S185875

53. Lim SB, Tan SJ, Lim WT, Lim CT. An extracellular matrix-related prognostic and predictive indicator for early-stage non-small cell lung cancer. Nature communications. 
644 2017;8(1):1734. http://dx.doi.org/10.1038/s41467-017-01430-6

645 54. Zhang X, Wang Y. Identification of hub genes and key pathways associated with the 646 progression of gynecological cancer. Oncology letters. 2019;18(6):6516-24. 647 http://dx.doi.org/10.3892/ol.2019.11004

648 55. Zhao H, Langerød A, Ji Y, Nowels KW, Nesland JM, Tibshirani R, et al. Different gene 649 expression patterns in invasive lobular and ductal carcinomas of the breast. Molecular biology of 650 the cell. 2004;15(6):2523-36. http://dx.doi.org/10.1091/mbc.e03-11-0786

651 56. Jiang Z, Zhu J, Ma Y, Hong C, Xiao S, Jin L. Tyrosylprotein sulfotransferase 1 expression 652 is negatively correlated with c-Met and lymph node metastasis in human lung cancer. Molecular medicine reports. 2015;12(4):5217-22. http://dx.doi.org/10.3892/mmr.2015.4096

654 57. Hollinger S, Hepler JR. Cellular regulation of RGS proteins: modulators and integrators of 655 G protein signaling. Pharmacological reviews. 2002;54(3):527-59. 656 http://dx.doi.org/10.1124/pr.54.3.527

657 58. Moratz C, Harrison K, Kehrl JH. Regulation of chemokine-induced lymphocyte migration 658 by RGS proteins. Methods in enzymology. 2004;389:15-32. http://dx.doi.org/10.1016/s0076$659 \quad 6879(04) 89002-5$

660 59. Gabrilovich D. Mechanisms and functional significance of tumour-induced dendritic-cell 661 defects. Nature reviews Immunology. 2004;4(12):941-52. http://dx.doi.org/10.1038/nri1498

662 60. Mariathasan S, Turley SJ, Nickles D, Castiglioni A, Yuen K, Wang Y, et al. TGF $\beta$ 663 attenuates tumour response to PD-L1 blockade by contributing to exclusion of T cells. Nature. 664 2018;554(7693):544-8. http://dx.doi.org/10.1038/nature25501

665 61. Garg M, Maurya N. WNT/ $\beta$-catenin signaling in urothelial carcinoma of bladder. World 666 journal of nephrology. 2019;8(5):83-94. http://dx.doi.org/10.5527/wjn.v8.i5.83

667 62. Spranger S, Bao R, Gajewski TF. Melanoma-intrinsic $\beta$-catenin signalling prevents anti668 tumour immunity. Nature. 2015;523(7559):231-5. http://dx.doi.org/10.1038/nature14404

669 63. Galon J, Bruni D. Approaches to treat immune hot, altered and cold tumours with 670 combination immunotherapies. Nature reviews Drug discovery. 2019;18(3):197-218. 671 http://dx.doi.org/10.1038/s41573-018-0007-y

672 64. Kato S, Weng QY, Insco ML, Chen KY, Muralidhar S, Pozniak J, et al. Gain-of-Function 673 Genetic Alterations of G9a Drive Oncogenesis. Cancer discovery. 2020;10(7):980-97. 674 http://dx.doi.org/10.1158/2159-8290.Cd-19-0532

675 65. Keam SP, Halse H, Nguyen T, Wang M, Van Kooten Losio N, Mitchell C, et al. High dose676 rate brachytherapy of localized prostate cancer converts tumors from cold to hot. Journal for immunotherapy of cancer. 2020;8(1). http://dx.doi.org/10.1136/jitc-2020-000792

678 66. Grauel AL, Nguyen B, Ruddy D, Laszewski T, Schwartz S, Chang J, et al. TGF $\beta$-blockade 679 uncovers stromal plasticity in tumors by revealing the existence of a subset of interferon-licensed 680 fibroblasts. Nature communications. 2020;11(1):6315. http://dx.doi.org/10.1038/s41467-020$681 \quad \underline{19920-5}$

682

\section{Figure legends}

684 Figure 1 Flow diagram for the present systematic analysis and validation.

685 Figure 2 The process of screening candidate genes. (A) Volcano plot of DEGs in GSE3167. 686 (B) Volcano plot of DEGs in GSE13507. (C) Volcano plot of DEGs in GSE37815. (D) Volcano 
687 plot of DEGs in GSE40355. (E) Volcano plot of DEGs in GSE121711. (F) Heatmap of top 20

688 upregulated and downregulated DEGs using robust rank aggregation analysis.

689 Figure 3 Evaluation of the risk score formula in the training (TCGA-BLCA) and validation

690 (GSE13507) sets. (A) Survival analysis between signature-defined risk groups. Patients in the

691 TCGA dataset were stratified into high- and low-risk groups according to the optimal cut-off

692 values. (B) Time-dependent ROC curves for 1-, 3-, and 5-year OS predictions in TCGA dataset.

693 (C) Survival analysis between signature-defined risk groups in GSE13507. (D) Time-dependent

694 ROC curves for 1-, 3-, and 5-year OS predictions in GSE13507. (E-F) Univariable and

695 multivariable analyses of risk score, age, gender, pathologic stage, T/N/M stage and molecular

696 subtype.

697 Figure 4 Validation of the nomogram for OS prediction in bladder cancer using the TCGA

698 dataset. (A) Nomogram predicting 1-, 3-, and 5-year OS. (B) Time-dependent ROC for 1-, 3-,

699 and 5-year OS predictions using the nomogram. (C) Calibration plot of the nomogram. (D) DCA

700 evaluation of the clinical utility of the nomogram.

701 Figure 5 Functional enrichment analysis of the eight-gene signature. (A) GO enrichment

702 analysis of the gene signature. (B) KEGG pathway enrichment analysis of the gene signature.

703 (C) Biological processes enriched in the high-risk group using GSEA.

704 Figure 6 Tumor immune landscape based on the gene signature. (A) The vioplot of 22

705 subpopulations of immune cells infiltration between high- and low-risk BC patients. (B) The

706 corHeatmap for all 22 immune cell proportions. (C) ssGSEA analysis revealed the enrichment of

707 immune-related functions, immune-related pathways and immune related cells between two risk

708 groups. (D) Chord plot of the correlation between gene signature riskscore and immune related

709 molecules.

710 Figure 7 Differential putative immunotherapeutic response prediction and verification of

711 tumor immune landscape in IMvigor210 cohort. (A) Submap analysis of immunotherapeutic

712 responses to anti-CTLA-4 and anti-PD-1 treatments in low- and high-risk group. (B) Survival

713 analysis between signature-defined risk groups. Patients in IMvigor210 cohort were stratified

714 into high- and low-risk groups according to the optimal cut-off values. (C) The violin plot of 22

715 subpopulations of immune cells infiltration between high- and low-risk groups in IMvigor 210

716 cohort. (D) The corHeatmap for all 22 immune cell proportions. (E) Exploration of tumor

717 microenvironment between two risk groups in IMvigor210 cohort using ssGSEA analysis (F)

718 Correlation between gene signature risk score and immune checkpoint molecules.

719 Figure 8 Risk score of novel eight-gene signature in six bladder cancer molecular subtypes. 


\section{Table $\mathbf{1}$ (on next page)}

Clinicopathological characteristics of bladder cancer patients in the TCGA cohort 
1 Table 1. Clinicopathological characteristics of bladder cancer patients in the TCGA cohort.

\begin{tabular}{|c|c|c|c|c|}
\hline Characteristics & All Patients & Low Risk & High Risk & $\mathrm{p}$-value \\
\hline Patients, no. (\%) & $392(100)$ & $195(49.7)$ & $197(50.3)$ & $\square$ \\
\hline T stage, no. $(\%)$ & $\square$ & $\square$ & $\square$ & 0.005 \\
\hline $\mathrm{T} 1+\mathrm{T} 2$ & $116(29.6)$ & $72(36.9)$ & $44(22.3)$ & $\square$ \\
\hline $\mathrm{T} 3$ & $189(48.2)$ & $90(46.2)$ & $99(50.3)$ & $\square$ \\
\hline $\mathrm{T} 4$ & $57(14.5)$ & $20(10.3)$ & $37(18.8)$ & $\square$ \\
\hline Unknown & $30(7.7)$ & $13(6.6)$ & $17(8.6)$ & $\square$ \\
\hline N stage, no. (\%) & $\square$ & $\square$ & $\square$ & 0.016 \\
\hline No & 227 (57.9) & $125(64.1)$ & $102(51.8)$ & $\square$ \\
\hline N1 & $125(31.9)$ & $49(25.1)$ & $76(38.5)$ & $\square$ \\
\hline Unknown & $40(10.2)$ & $21(10.8)$ & $19(9.7)$ & $\square$ \\
\hline M stage, no. (\%) & $\square$ & $\square$ & $\square$ & 0.024 \\
\hline M0 & $187(47.7)$ & $104(53.4)$ & $83(42.1)$ & $\square$ \\
\hline M1 & $10(2.6)$ & $2(1.0)$ & $8(4.1)$ & $\square$ \\
\hline Unknown & $195(49.7)$ & $89(45.6)$ & $106(53.8)$ & $\square$ \\
\hline Stage, no. $(\%)$ & $\square$ & $\square$ & $\square$ & 0.002 \\
\hline Stage I+II & $125(31.9)$ & $76(39.0)$ & $49(24.9)$ & $\square$ \\
\hline
\end{tabular}




\begin{tabular}{|c|c|c|c|c|c|}
\hline Stage III & 137 (34.9) & 68 (34.9) & $69(35.0)$ & $\square$ & \\
\hline Stage IV & $130(33.2)$ & $51(26.1)$ & $79(40.1)$ & $\square$ & \\
\hline Grade, no. (\%) & $\square$ & $\square$ & $\square$ & & $<0.001$ \\
\hline Low Grade & $18(4.6)$ & $18(9.2)$ & $0(0)$ & & $\sqsubset$ \\
\hline High Grade & $372(94.9)$ & $176(90.3)$ & $196(99.5)$ & & $\sqsubset$ \\
\hline Unknown & $2(0.5)$ & $1(0.5)$ & $1(0.5)$ & & $\sqsubset$ \\
\hline Survival status, no. (\%) & $\square$ & $\square$ & $\square$ & & $<0.001$ \\
\hline Alive & $241(61.5)$ & $146(74.9)$ & $95(48.2)$ & $\square$ & \\
\hline Dead & $151(38.5)$ & $49(25.1)$ & $102(51.8)$ & $\square$ & \\
\hline Median months & 64.7 & 72.8 & 56.9 & $\square$ & \\
\hline
\end{tabular}


Figure 1

\section{Flow diagram for the present systematic analysis and validation}

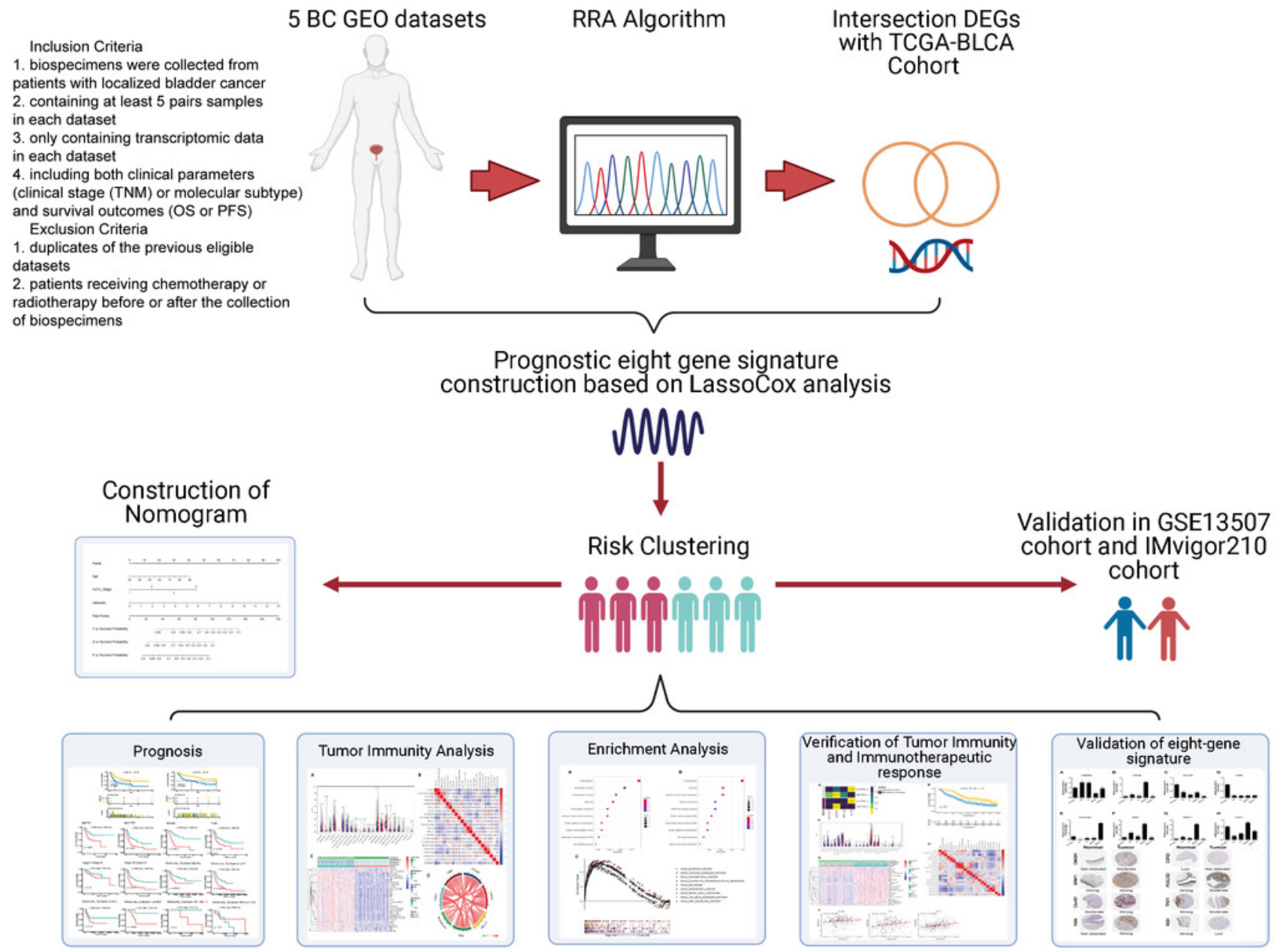


Figure 2

The process of screening candidate genes

(A) Volcano plot of DEGs in GSE3167. (B) Volcano plot of DEGs in GSE13507. (C) Volcano plot of DEGs in GSE37815. (D) Volcano plot of DEGs in GSE40355. (E) Volcano plot of DEGs in GSE121711. (F) Heatmap of top 20 upregulated and downregulated DEGs using robust rank aggregation analysis 

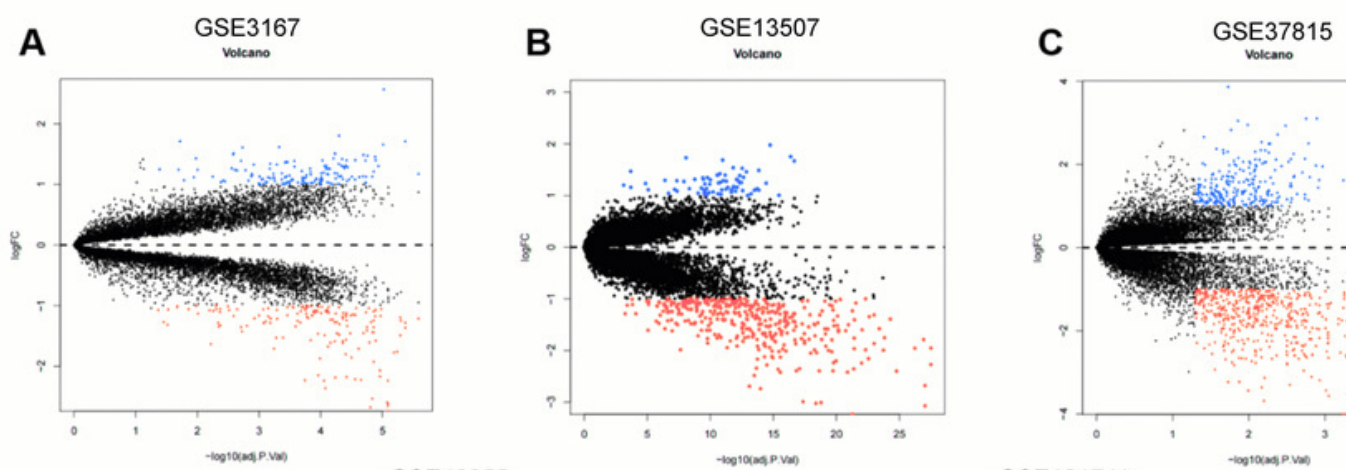
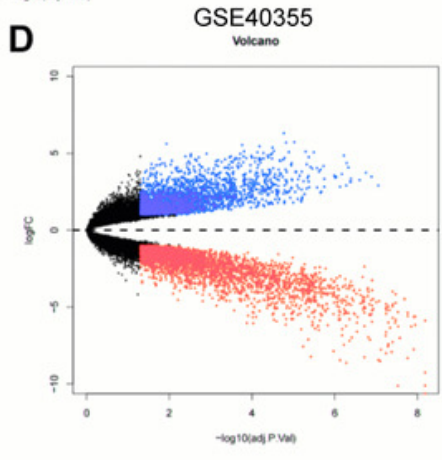

$\mathbf{F}$

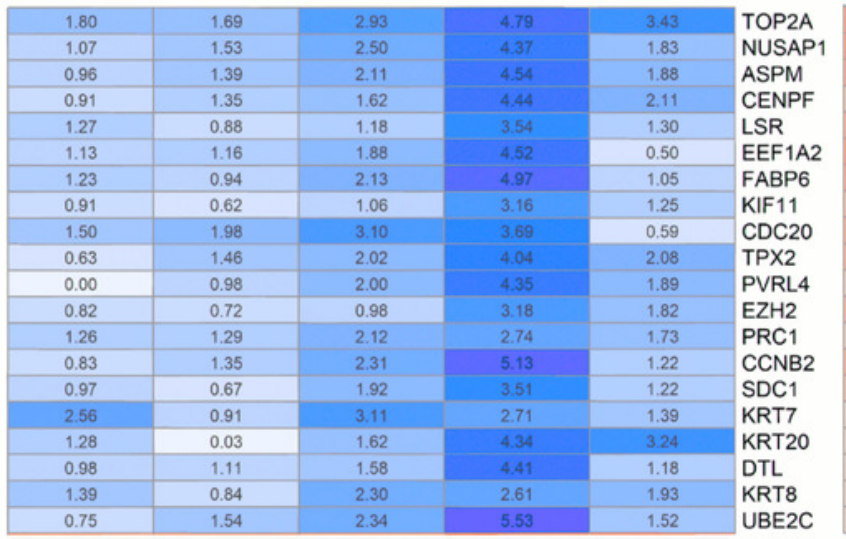

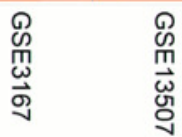

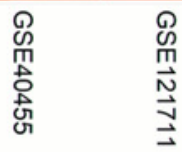
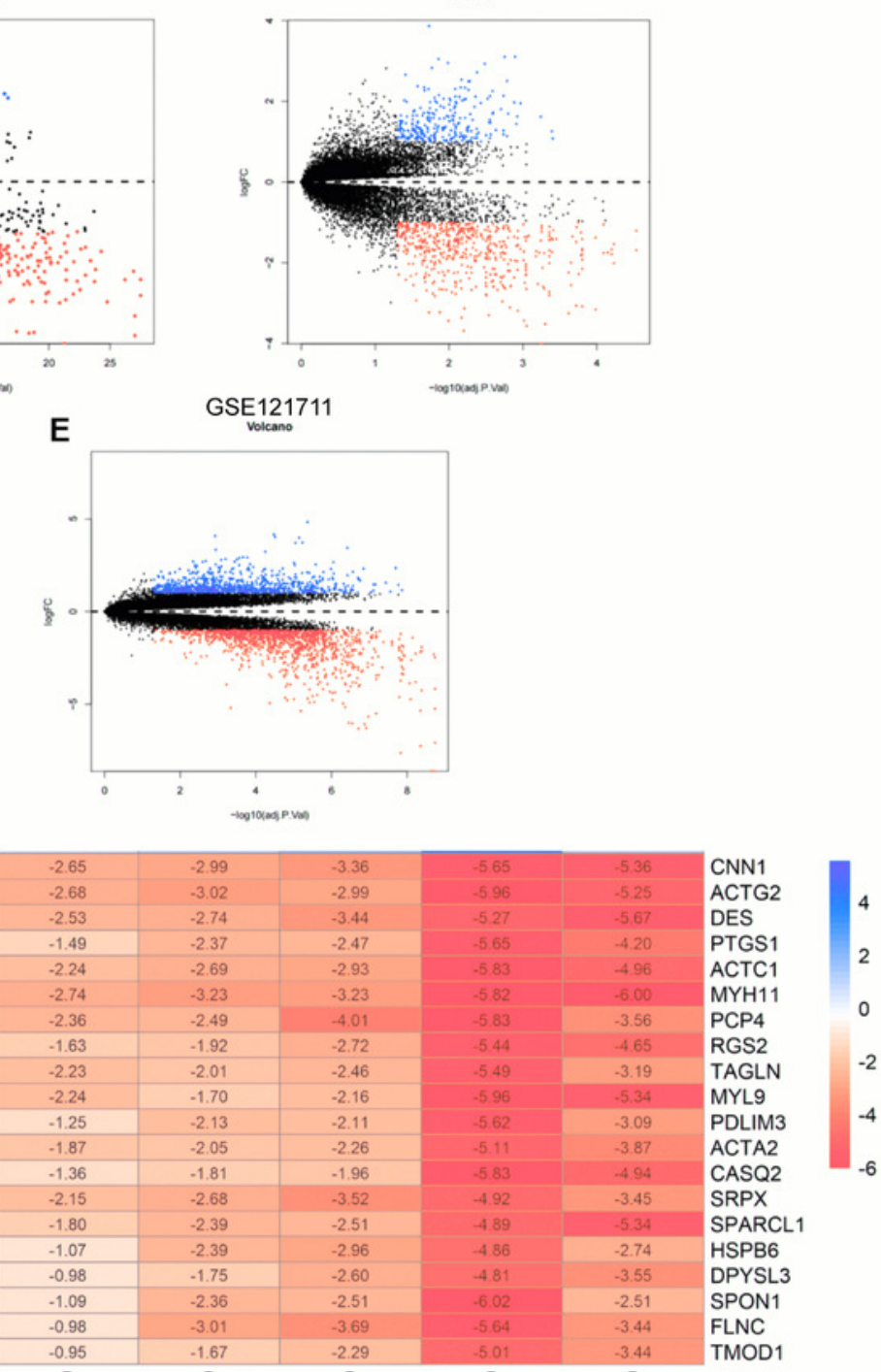

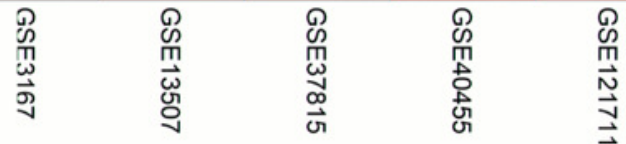




\section{Figure 3}

Evaluation of the risk score formula in the training (TCGA-BLCA) and validation (GSE13507) sets

(A) Survival analysis between signature-defined risk groups. Patients in the TCGA dataset were stratified into high- and low-risk groups according to the optimal cut-off values. (B) Time-dependent ROC curves for 1-, 3-, and 5-year OS predictions in TCGA dataset. (C) Survival analysis between signature-defined risk groups in GSE13507. (D) Time-dependent ROC curves for 1-, 3-, and 5-year OS predictions in GSE13507. (E-F) Univariable and multivariable analyses of risk score, age, gender, pathologic stage, T/N/M stage and molecular subtype 
A
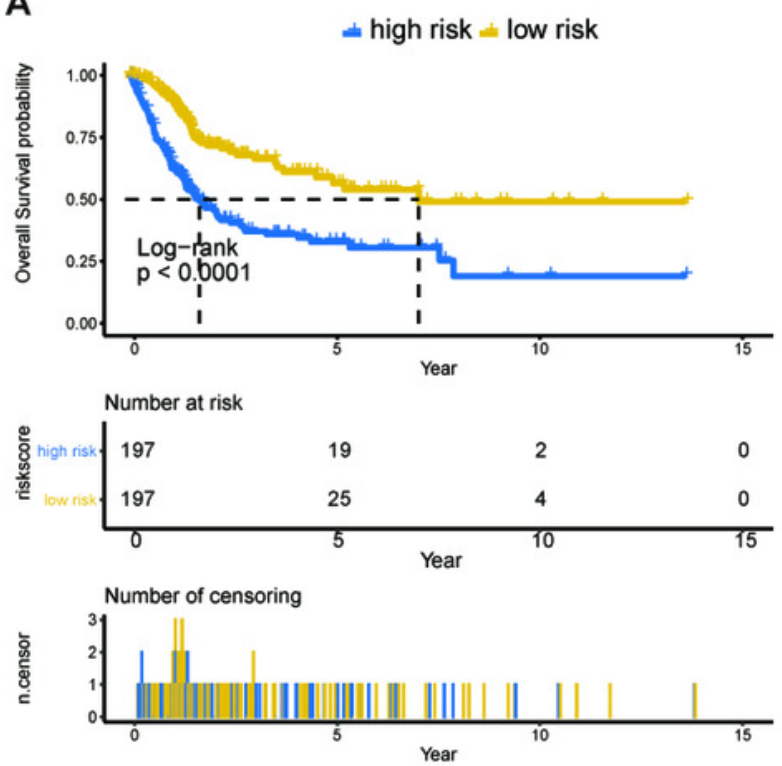

C

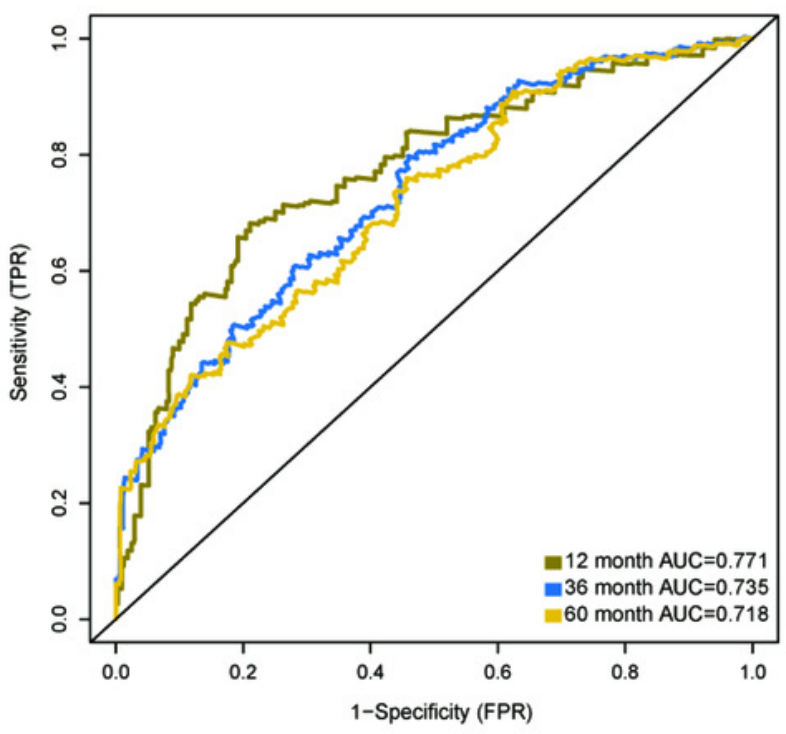

E

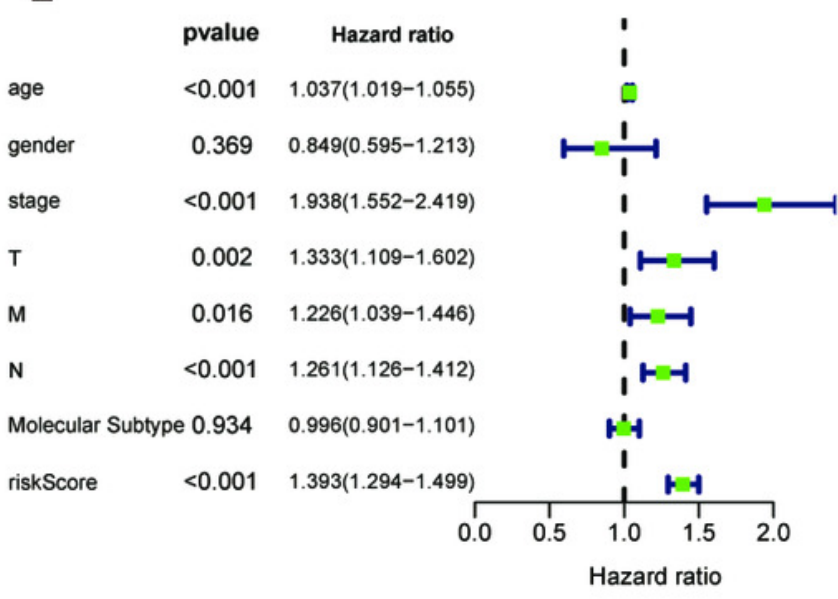

B
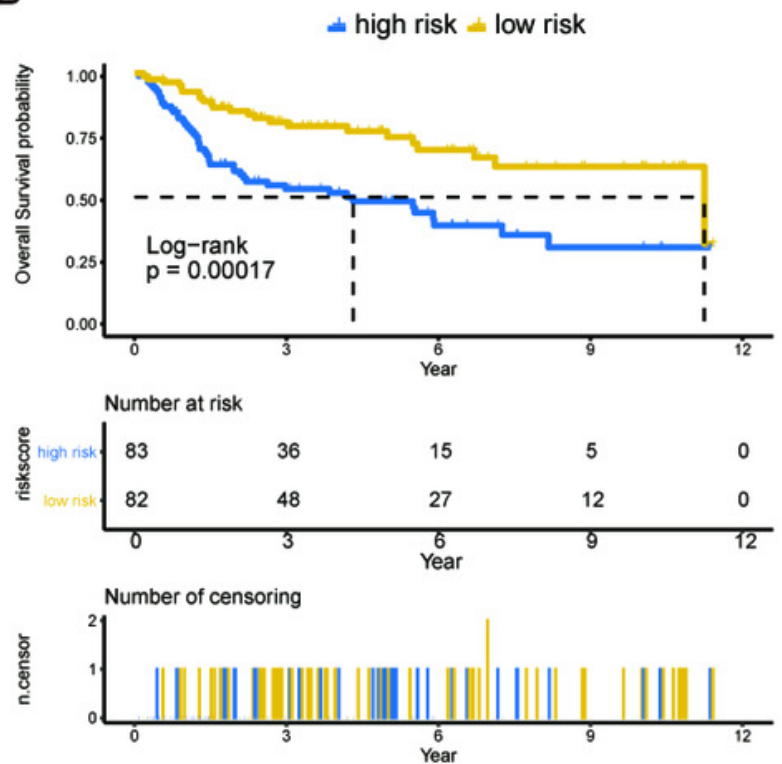

D

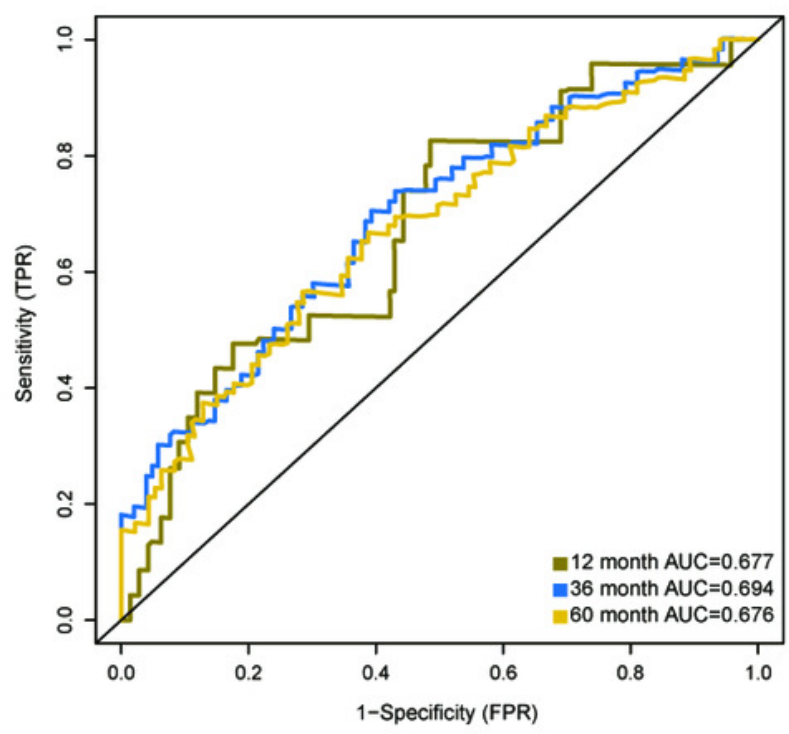

$\mathbf{F}$

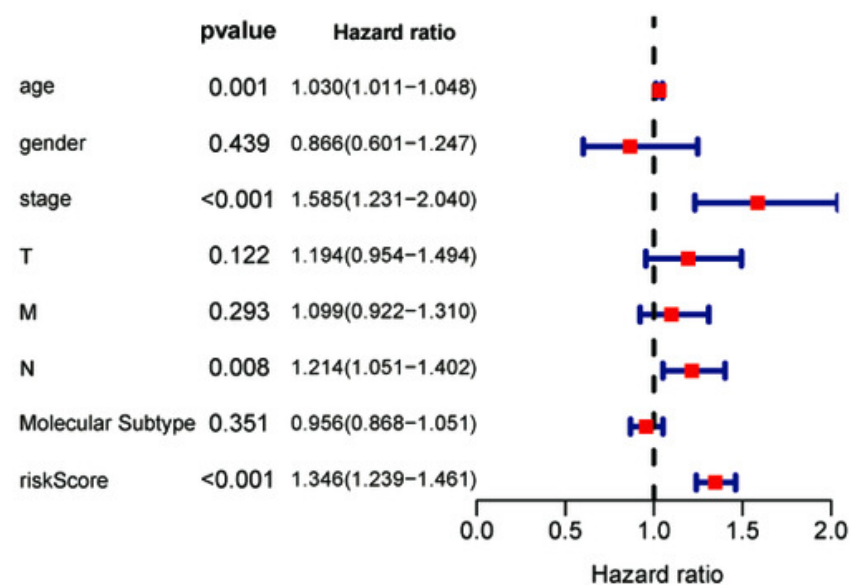


Figure 4

Validation of the nomogram for OS prediction in bladder cancer using the TCGA dataset

(A) Nomogram predicting 1-, 3-, and 5-year OS. (B) Time-dependent ROC for 1-, 3-, and 5year OS predictions using the nomogram. (C) Calibration plot of the nomogram. (D) DCA evaluation of the clinical utility of the nomogram

A
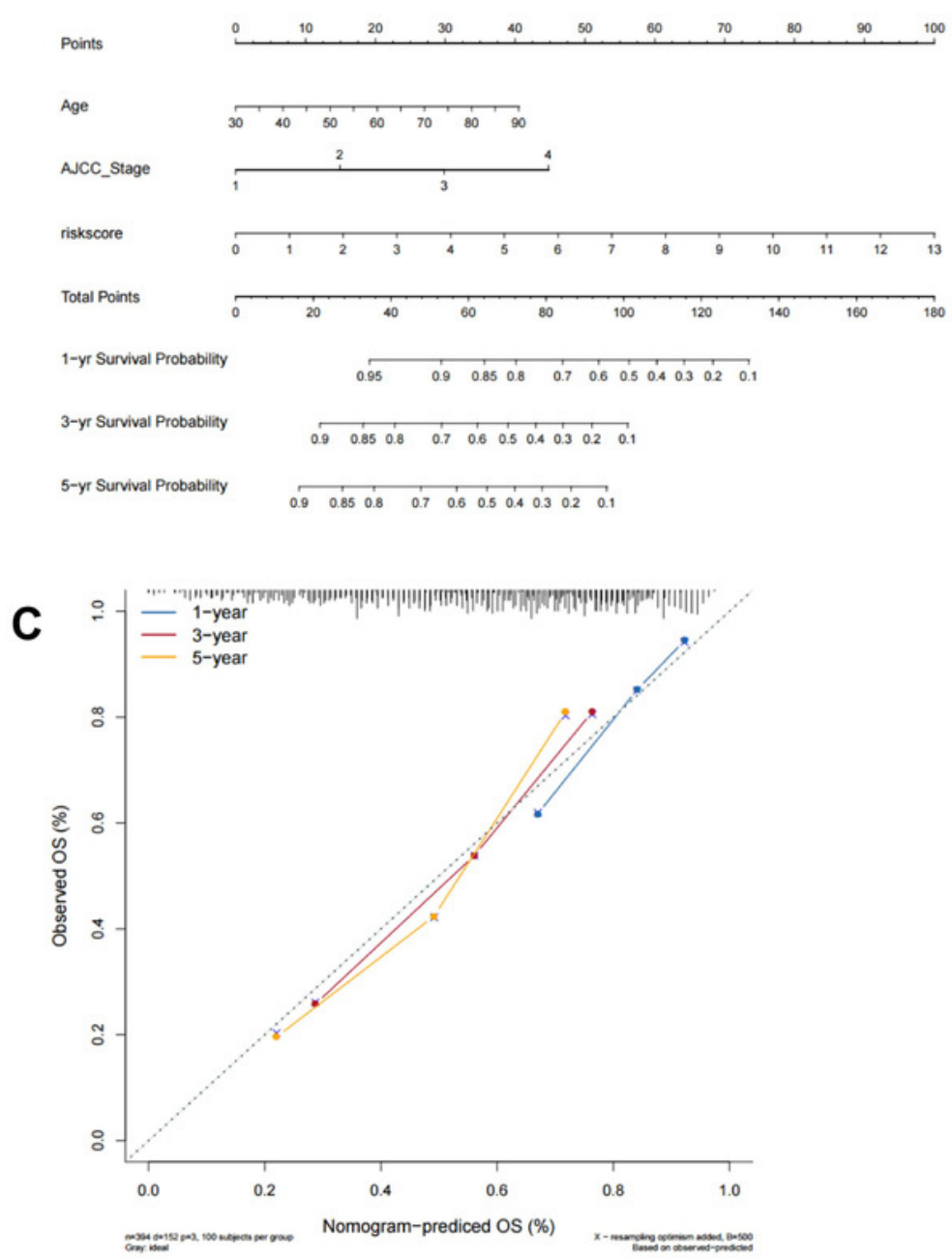

B

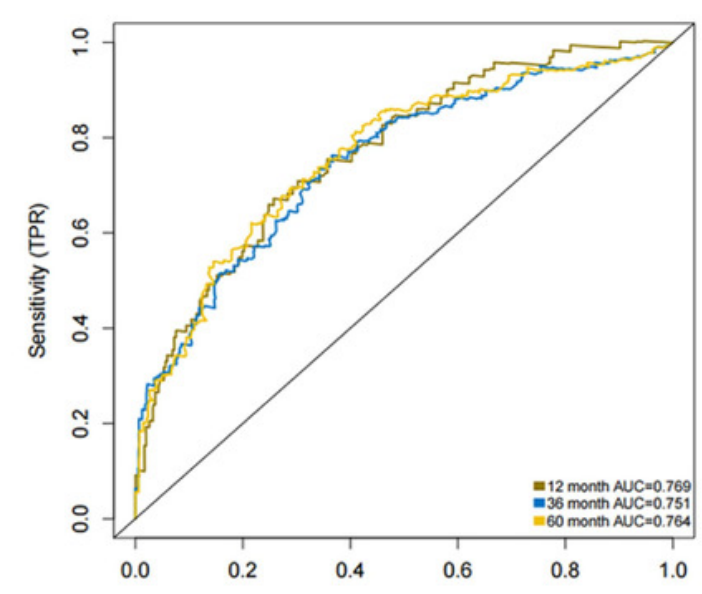

D

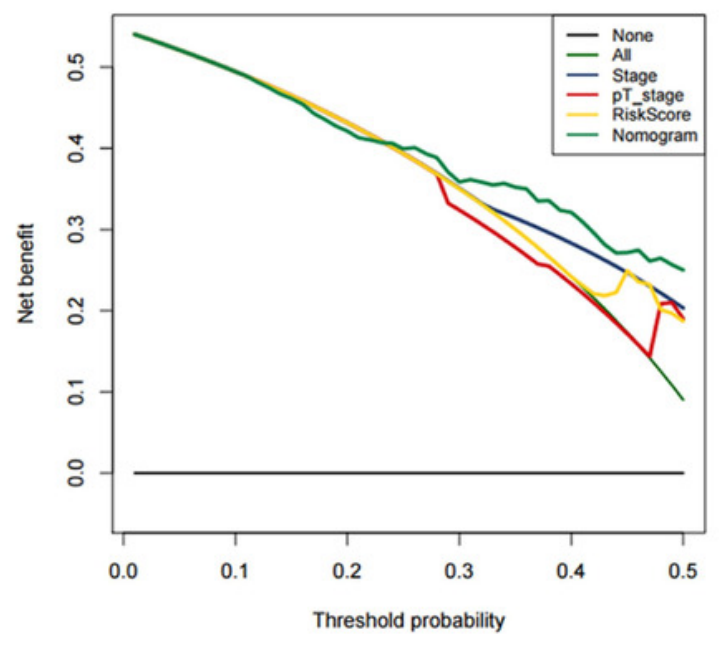


Figure 5

Functional enrichment analysis of the eight-gene signature

(A) GO enrichment analysis of the gene signature. (B) KEGG pathway enrichment analysis of the gene signature. (C) Biological processes enriched in the high-risk group using GSEA 
A

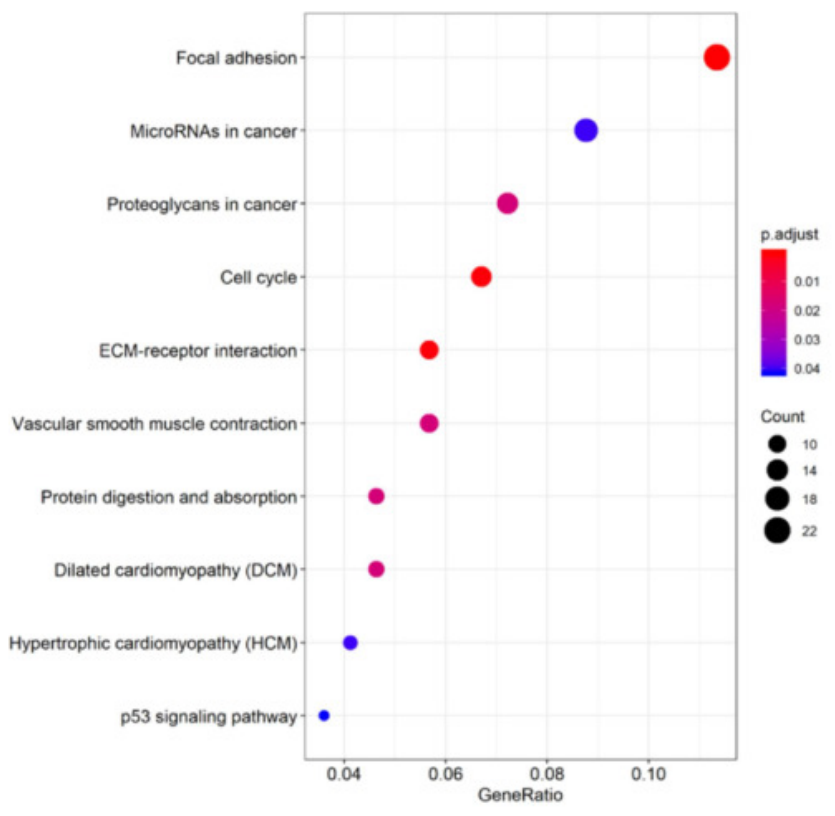

C

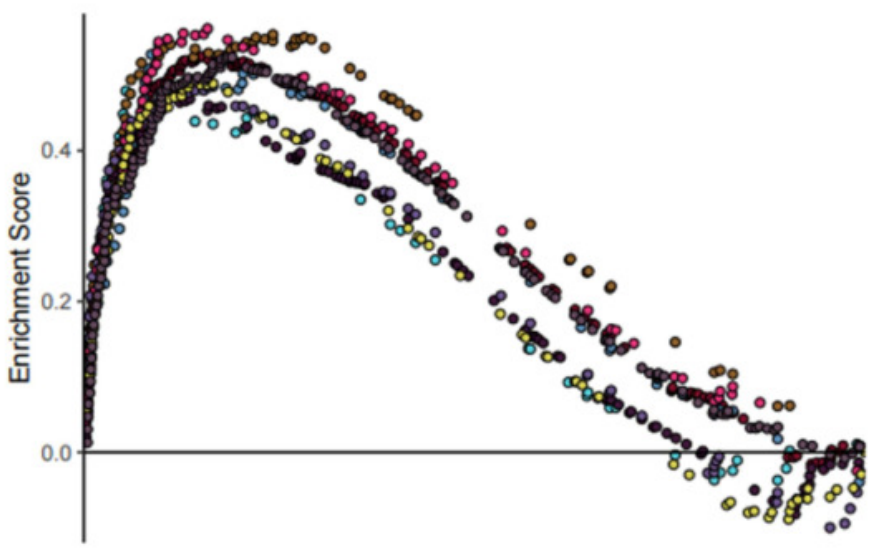

B

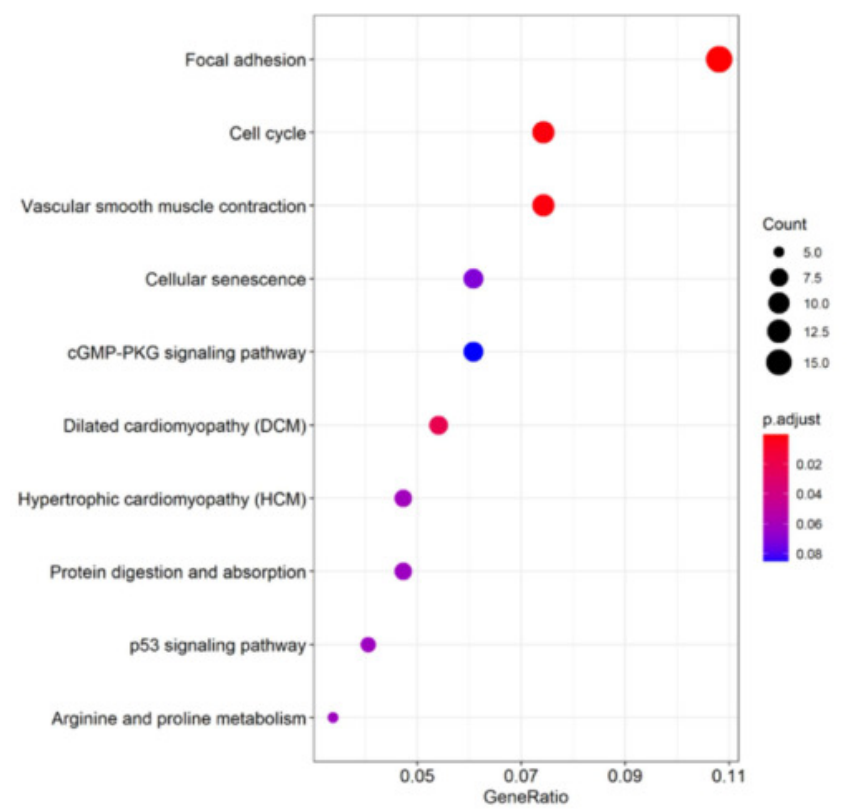

- KEGG_BLADDER_CANCER

- KEgg_Calcium_Signaling_pathway

- KEgG_ENDOMETRIAL_CANCER

- KEgG_leukocyte_transendothelial_migRATION

- KEgG_MELANOMA

- KEgG_PANCREATIC_CANCER

- KEgG_RENAL_CELL_CARCINOMA

- KEGG_tGF_BETA_SIGNALING_PATHWAY

- KEgG_WNT_SIGNALING_PATHWAY

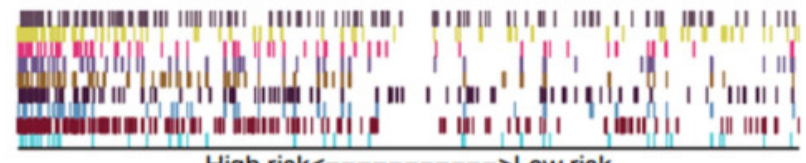

High risk<------------->Low risk 
Figure 6

Tumor immune landscape based on the gene signature

(A) The vioplot of 22 subpopulations of immune cells infiltration between high- and low-risk BC patients. (B) The corHeatmap for all 22 immune cell proportions. (C) ssGSEA analysis revealed the enrichment of immune-related functions, immune-related pathways and immune related cells between two risk groups. (D) Chord plot of the correlation between gene signature riskscore and immune related molecules

A
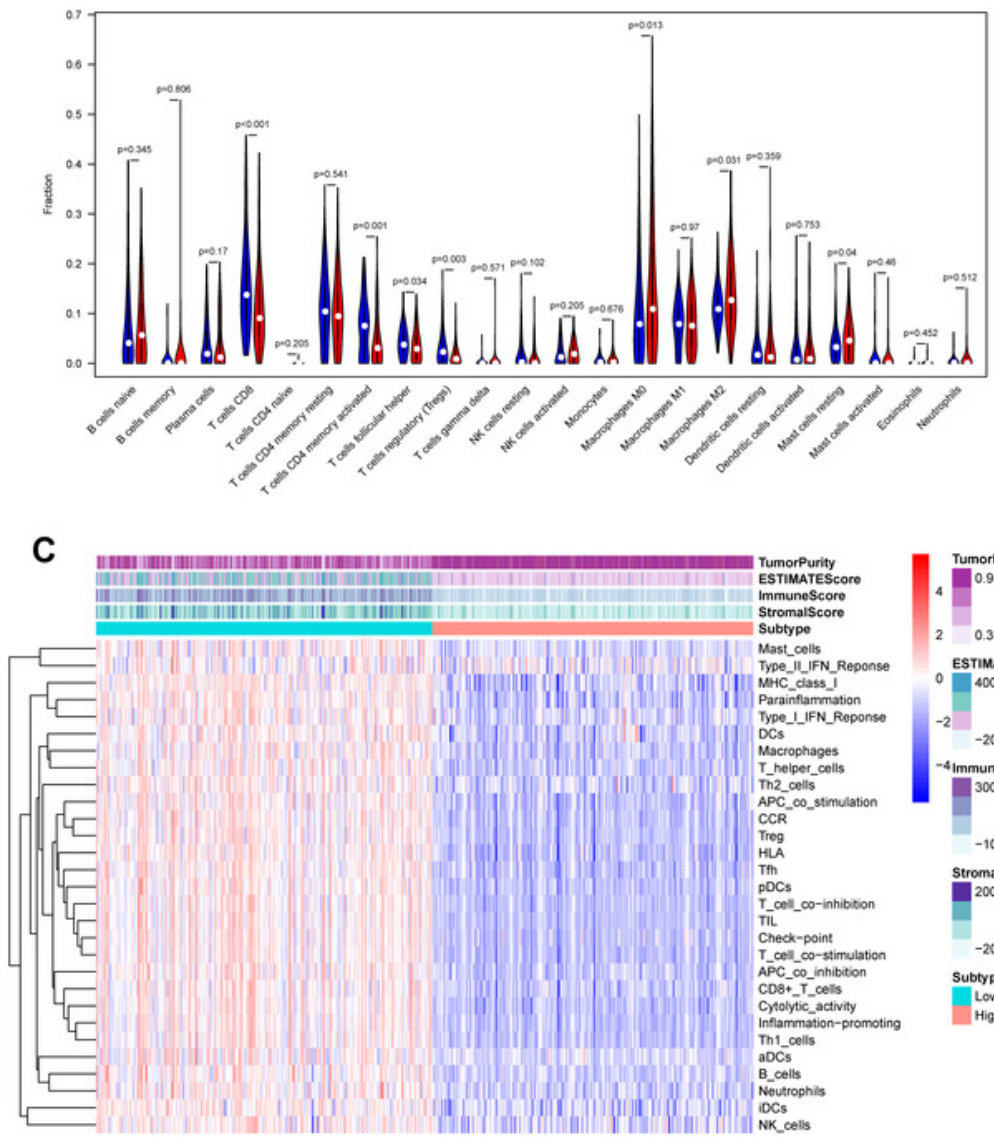

B

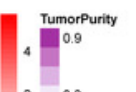
Estimatescoro D $-2-2000$

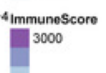

$-1000$ Stromalscore
2000 -2000
Subtype
Low Risk

B

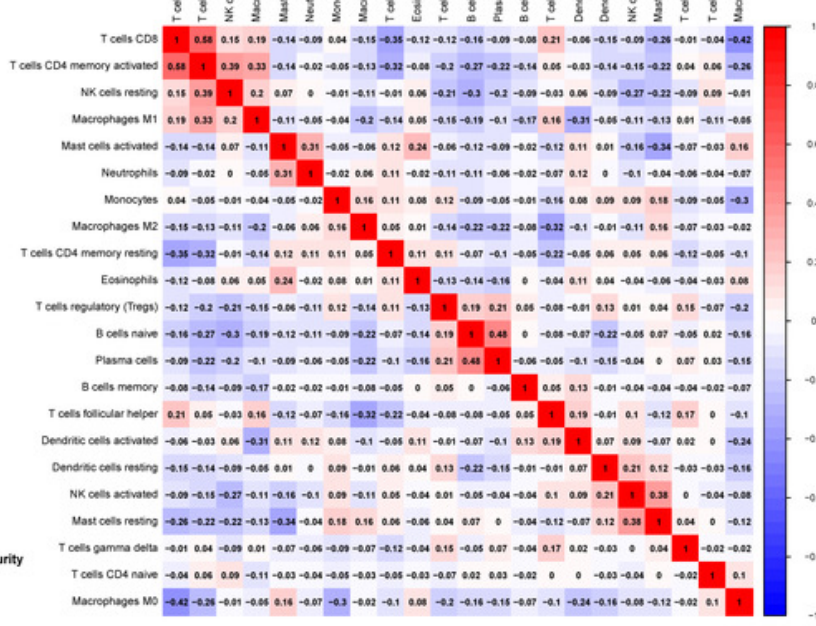

D

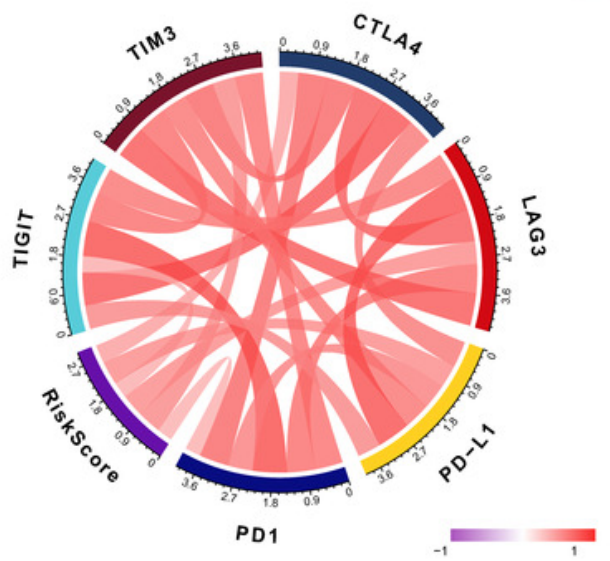




\section{Figure 7}

Differential putative immunotherapeutic response prediction and verification of tumor immune landscape in IMvigor210 cohort

(A) Submap analysis of immunotherapeutic responses to anti-CTLA-4 and anti-PD-1 treatments in low- and high-risk group. (B) Survival analysis between signature-defined risk groups. Patients in IMvigor210 cohort were stratified into high- and low-risk groups according to the optimal cut-off values. (C) The violin plot of 22 subpopulations of immune cells infiltration between high- and low-risk groups in IMvigor210 cohort. (D) The corHeatmap for all 22 immune cell proportions. (E) Exploration of tumor microenvironment between two risk groups in IMvigor210 cohort using SSGSEA analysis (F) Correlation between gene signature risk score and immune checkpoint molecules 
A
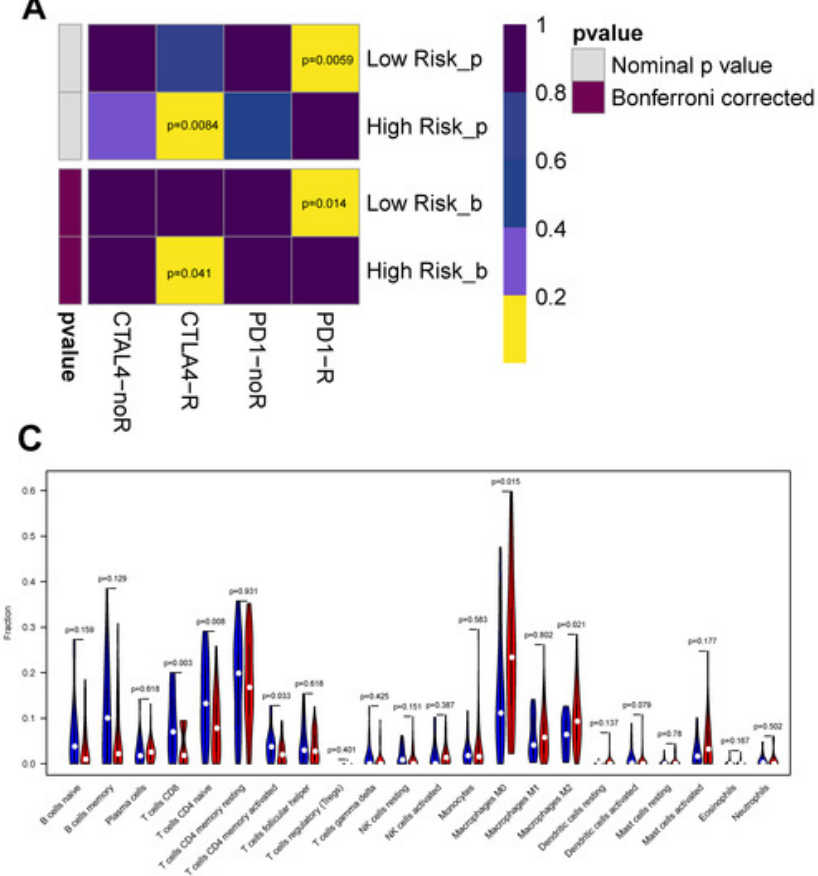

\section{E}

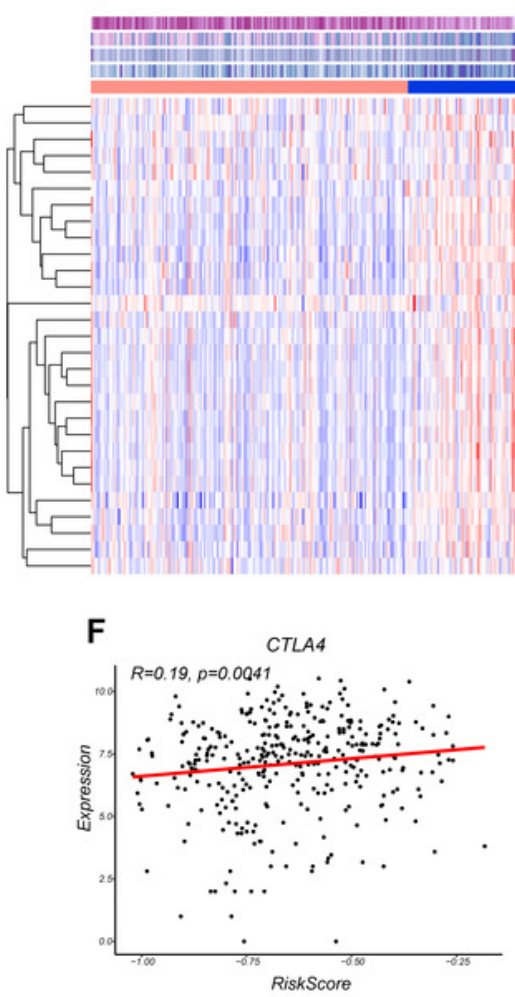

B

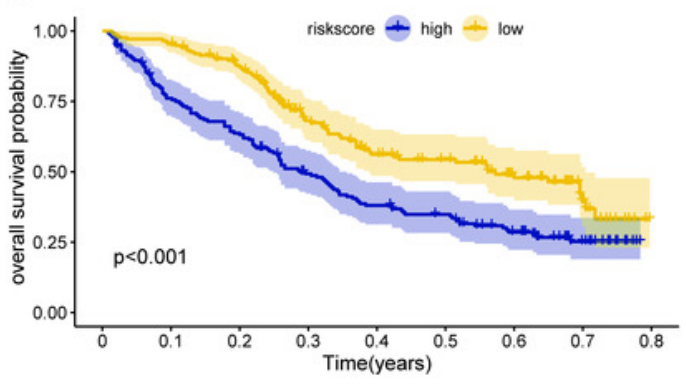

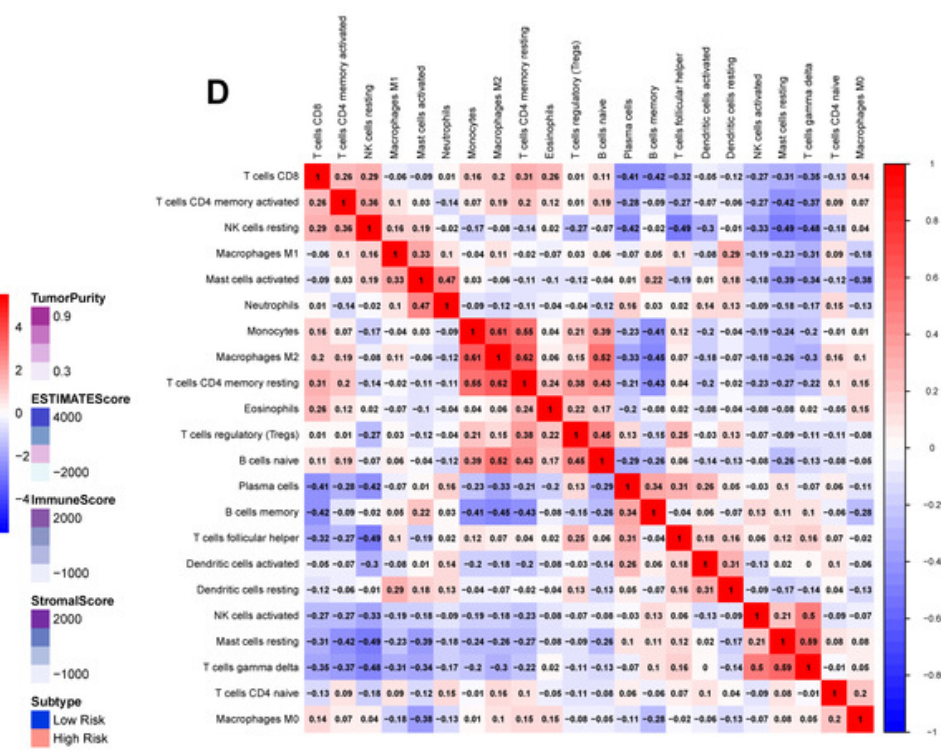
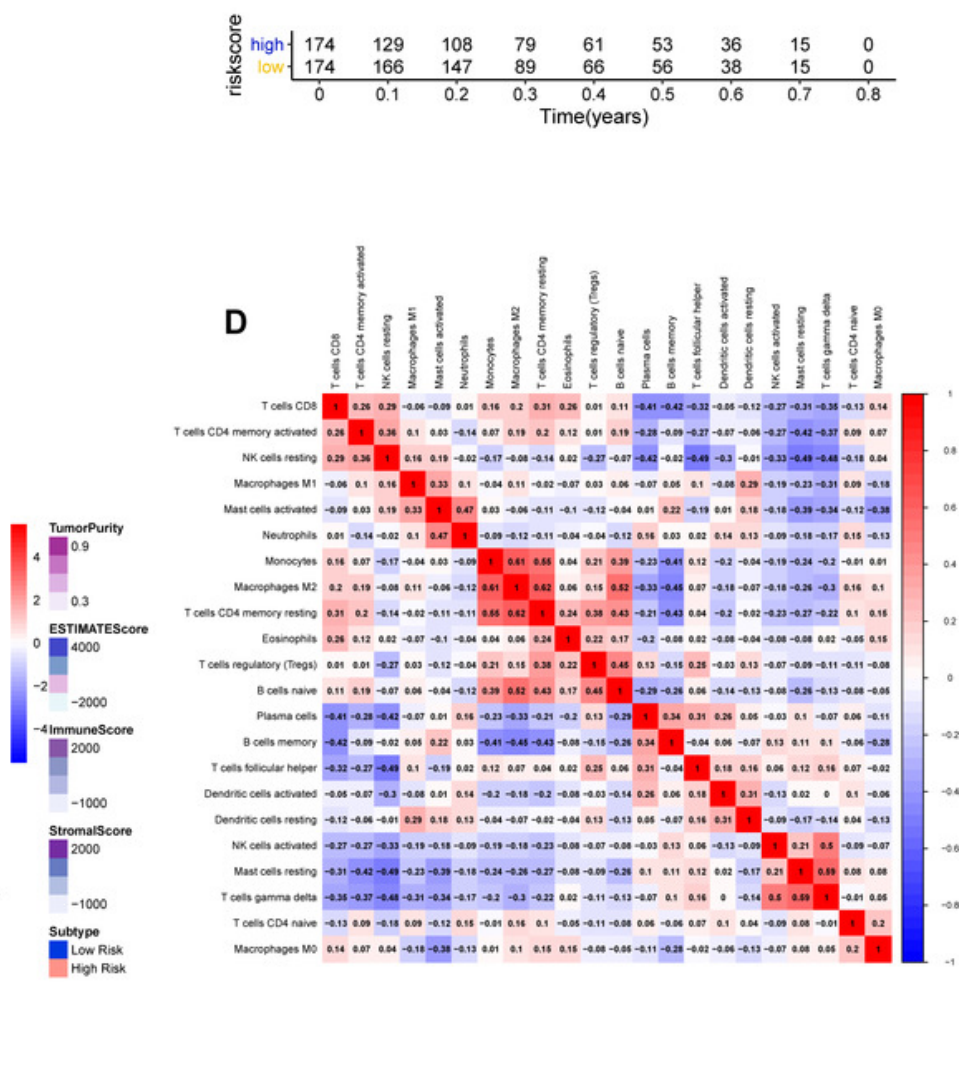
Figure 8

Risk score of novel eight-gene signature in six bladder cancer molecular subtypes

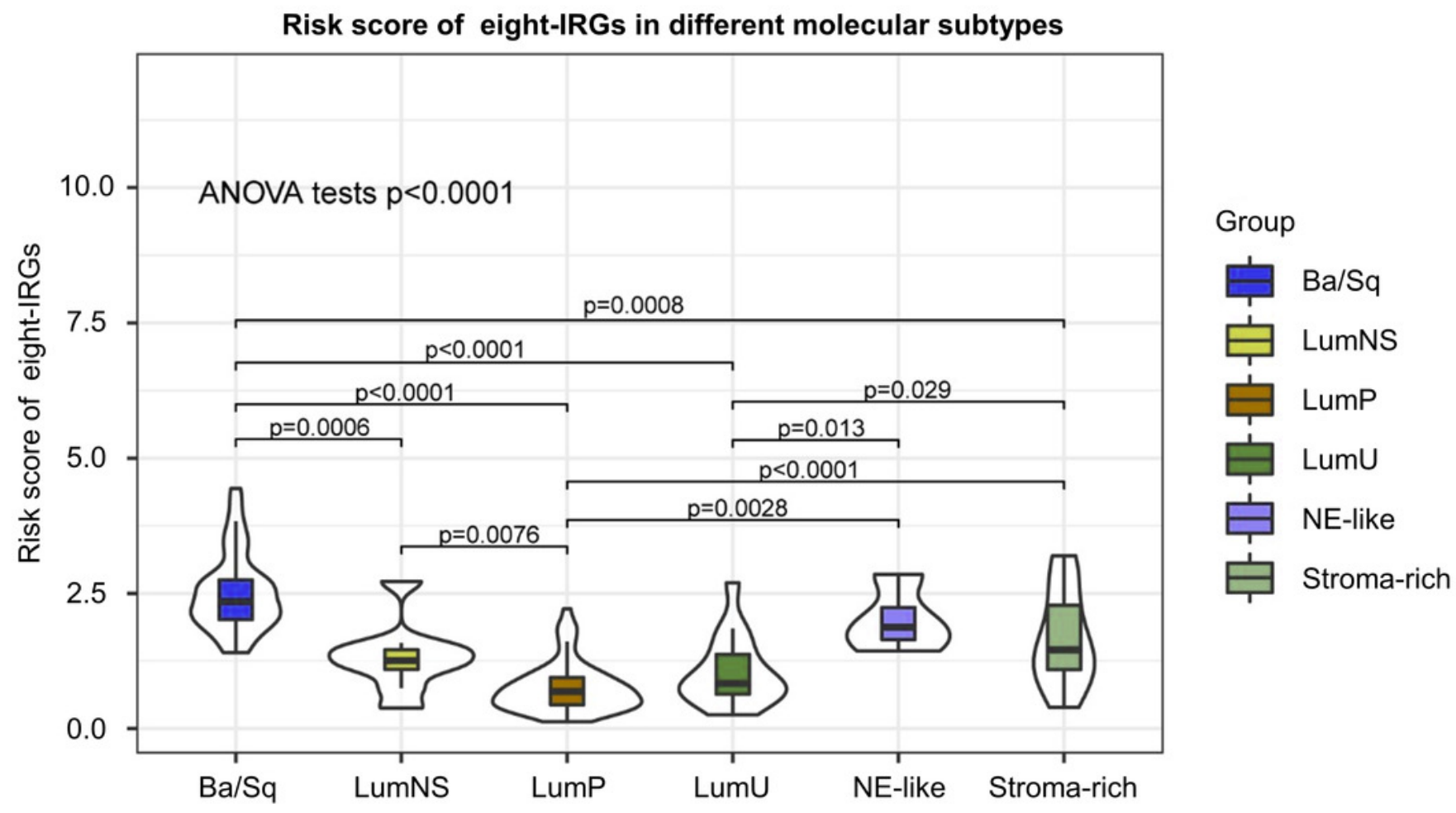

